Pacific

Journal of

Mathematics

METRICAL DIOPHANTINE ANALYSIS FOR TAME PARABOLIC ITERATED FUNCTION SYSTEMS

Bernd O. Stratmann and Mariusz Urbański 


\title{
METRICAL DIOPHANTINE ANALYSIS FOR TAME PARABOLIC ITERATED FUNCTION SYSTEMS
}

\author{
Bernd O. Stratmann and Mariusz Urbański
}

\begin{abstract}
We study various aspects of tame finite parabolic iterated function systems that satisfy a certain open set condition. The first goal in our analysis of these systems is a detailed investigation of the conformal measure on the associated limit sets. We derive a formula that describes in a uniform way the scaling of this measure at arbitrary limit points. The second goal is to provide a metrical Diophantine analysis for these parabolic limit sets in the spirit of theorems of Jarník and Khintchine in number theory. Subsequently, we show that this Diophantine analysis gives rise to refinements of the description of the conformal measure in terms of Hausdorff and packing measures with respect to certain gauge functions.
\end{abstract}

\section{Introduction}

For a large class of fractal sets the idea of an iterated function system has turned out to be a very convenient and efficient concept. Traditionally, the development of fractal geometry was always very much inspired by various phenomena that appear in conformal analysis and number theory. In this paper we continue this tradition by studying metrical Diophantine aspects of certain tame parabolic iterated function systems. This study generalizes results for geometrically finite Kleinian groups with parabolic elements (obtained in $[\mathbf{S 1}],[\mathbf{S 2}],[\mathbf{S 3}],[\mathbf{S V}]$, see also $[\mathbf{H V}],[\mathbf{S u}]$ ) and for parabolic rational rational functions (obtained in [SU1], [SU2]), which represent complex analytic analogues of Jarník's number theoretical theorem on well-approximable numbers $([\mathbf{J}],[\mathbf{B}])$ and Khintchine's on a qualitative description of the 'essential support' of the 1-dimensional Lebesgue measure $([\mathbf{K}])$.

The paper is organized as follows: in Section 2 we first define the class of tame finite parabolic iterated function systems that satisfy the Super Strong Open Set Condition (SSOSC). We then recall a few immediate geometrical implications of the bounded distortion properties. In Section 3 we study the $h$-conformal measures arising from these parabolic systems. (Here, $h$ denotes the Hausdorff dimension of the limit set associated to such a system.) We obtain a formula that describes in a uniform way the scaling of this measure 
at arbitrary elements of the limit set. As a by-product we obtain an estimate on the local behaviour of the $h$-conformal measure at parabolic points. In Section 4 we analyse the limit sets from a Diophantine point of view. Our general approach here follows roughly the analysis given in $[\mathbf{S 1}],[\mathbf{S 2}],[\mathbf{S V}]$, [SU1], [SU2]. Nevertheless, the construction of the main tool, namely the measure $\mu$ on a Cantor-like subset of the limit set, is different. This construction is simplified and its geometrical and dynamical significance is clarified. Finally, we establish various limit laws leading up to the Khintchine Limit Law for tame parabolic iterated function systems. Subsequently, we show that these laws provide some efficient control on the fluctuations of the $h$-conformal measure, giving rise to refinements of the description of the $h$-conformal measure in terms of Hausdorff and packing measures with respect to some gauge functions.

\section{Preliminaries}

We begin by giving a description of our setting. Let $X$ be a compact subset of some Euclidean space $\mathbb{R}^{d}$ such that $X$ has nonempty interior and is contained in a bounded connected open set $V$. Suppose that there are countably many conformal maps $\phi_{i}: X \rightarrow X, i \in I$, with $I$ having at least two elements. Then the system $S=\left\{\phi_{i}: i \in I\right\}$ is called a conformal iterated function system if and only if the following eight conditions are satisfied:

(1) (Open Set Condition) $\phi_{i}(\operatorname{Int}(X)) \cap \phi_{j}(\operatorname{Int}(X))=\emptyset$ for all $i \neq j$.

(2) $\left|\phi_{i}^{\prime}(x)\right|<1$ everywhere except for finitely many pairs $\left(i, x_{i}\right), i \in I$, for which $x_{i}$ is the unique fixed-point of $\phi_{i}$ and $\left|\phi_{i}^{\prime}\left(x_{i}\right)\right|=1$. Such pairs and indices $i$ will be called parabolic and the set of parabolic indices will be denoted by $\Omega$. All other indices will be called hyperbolic.

(3) For all $n \geq 1, \omega=\left(\omega_{1}, \ldots, \omega_{n}\right) \in I^{n}$ we have that if $\omega_{n}$ is a hyperbolic index or if $\omega_{n-1} \neq \omega_{n}$, then $\phi_{\omega}$ admits a conformal extension to $V \subset \mathbb{R}^{d}$ that maps $V$ into itself.

(4) If $i$ is a parabolic index, then $\bigcap_{n \geq 0} \phi_{i^{n}}(X)=\left\{x_{i}\right\}$ (Hence in particular, the diameter of the set $\phi_{i^{n}}(X)$ tends to 0 for $n$ tending to infinity.)

(5) (Cone Condition) There exist $\alpha, l>0$ such that for every $x \in \partial X \subset \mathbb{R}^{d}$ there exists an open cone $\operatorname{Con}\left(x, u_{x}, \alpha, l\right) \subset \operatorname{Int}(X)$ with vertex $x$, $\left\|u_{x}\right\|=1$ and central angle $\alpha$. Here, we have set $\operatorname{Con}\left(x, u_{x}, \alpha, l\right):=$ $\left\{y: 0<\left(y-x, u_{x}\right) \leq \cos \alpha\|y-x\| \leq l\right\}$.

(6) There exists $0<s<1$ such that for all $n \geq 1, \omega \in I^{n}$ we have that if $\omega_{n}$ is a hyperbolic index or if $\omega_{n-1} \neq \omega_{n}$, then $\left\|\phi_{\omega}^{\prime}\right\| \leq s$.

(7) (Bounded Distortion Property) There exists $K \geq 1$ such that for all $n \geq 1, \omega=\left(\omega_{1}, \ldots, \omega_{n}\right) \in I^{n}$ and $x, y \in V$ we have that if $\omega_{n}$ is a hyperbolic index or if $\omega_{n-1} \neq \omega_{n}$, then

$$
\left|\phi_{\omega}^{\prime}(y)\right| \leq K\left|\phi_{\omega}^{\prime}(x)\right| .
$$


(8) There are constants $L \geq 1, \alpha>0$ such that

$$
\left\|\phi_{i}^{\prime}(y)|-| \phi_{i}^{\prime}(x)|| \leq L\right\| \phi_{i}^{\prime} \||y-x|^{\alpha} \quad \text { for all } i \in I \text { and } x, y \in V .
$$

Note that if $\Omega=\emptyset$, the system $S$ is called hyperbolic, and that if $\Omega \neq \emptyset$, then $S$ is called parabolic. Throughout this paper we shall always assume without further notice that the system $S$ is parabolic and the alphabet $I$ is finite.

We now state a few immediate geometrical consequences of the bounded distortion properties (7), (8) and the cone condition (5). For the proofs of these statements we refer to [MU1] and [MU3].

For all hyperbolic words $\omega \in I^{*}$ and all convex subsets $C$ of $V$ we have

$$
\operatorname{diam}\left(\phi_{\omega}(C)\right) \leq\left\|\phi_{\omega}^{\prime}\right\| \operatorname{diam}(C)
$$

and

$$
\operatorname{diam}\left(\phi_{\omega}(V)\right) \leq D\left\|\phi_{\omega}^{\prime}\right\| .
$$

Here, the norm $\|\cdot\|$ is the supremum norm on $V$, and $D \geq 1$ denotes a universal constant. Moreover, for every $x \in X, 0<r \leq \operatorname{Dist}(X, \partial V)$, and for every hyperbolic word $\omega \in I^{*}$ we have

$$
\operatorname{diam}\left(\phi_{\omega}(X)\right) \geq D^{-1}\left\|\phi_{\omega}^{\prime}\right\|
$$

and

$$
\phi_{\omega}(B(x, r)) \supset B\left(\phi_{\omega}(x), K^{-1}\left\|\phi_{\omega}^{\prime}\right\| r\right) .
$$

Also, there exists $0<\beta \leq \alpha$ such that for all $x \in X$ and for all hyperbolic words $\omega \in I^{*}$

$$
\phi_{\omega}(\operatorname{Int}(X)) \supset \operatorname{Con}\left(\phi_{\omega}(x), \beta, D^{-1}\left\|\phi_{\omega}^{\prime}\right\|\right) \supset \operatorname{Con}\left(\phi_{\omega}(x), \beta, D^{-2} \operatorname{diam} \phi_{\omega}(V)\right),
$$

where $\operatorname{Con}\left(\phi_{\omega}(x), \beta, D^{-1}\left\|\phi_{\omega}^{\prime}\right\|\right)$ and $\operatorname{Con}\left(\phi_{\omega}(x), \beta, D^{-2} \operatorname{diam}\left(\phi_{\omega}(V)\right)\right)$ denote some cones with vertices at $\phi_{\omega}(x)$, angles $\beta$, and altitudes $D^{-1}\left\|\phi_{\omega}^{\prime}\right\|$ and $D^{-2} \operatorname{diam}\left(\phi_{\omega}(V)\right)$ respectively. Finally, for every $\omega \in I^{*}$ (not necessarily hyperbolic) and every $x \in X$, there exists an altitude $l(\omega, x)>0$ such that

$$
\phi_{\omega}(\operatorname{Int}(X)) \supset \operatorname{Con}\left(\phi_{\omega}(x), \beta, l(\omega, x)\right) .
$$

We emphasize that for $d \geq 2$ the conditions (7) and (8) with $\alpha=1$ can be deduced from condition (3). For $d \geq 3$, this has been shown in [U1]. For $d=2$, conditions (7) and (8) follow from Koebe's distortion theorem combined with the observation that complex conjugation in $\mathbb{C}$ is an isometry.

Let $I^{*}$ denote the set of all finite words in the alphabet $I$, and let $I^{\infty}$ be the set of all infinite sequences with entries in $I$. By condition (3), we have $\phi_{\omega}(V) \subset V$, for every hyperbolic word $\omega$. For each $\omega \in I^{*} \cup I^{\infty}$, we define the length of $\omega$ by the uniquely determined relation $\omega \in I^{|\omega|}$. If $\omega \in I^{*} \cup I^{\infty}$ and $n \leq|\omega|$, then we write $\left.\omega\right|_{n}$ to denote the word $\omega_{1} \omega_{2} \ldots \omega_{n}$. In [MU1] 
it was shown that $\lim _{n \rightarrow \infty} \sup _{|\omega|=n}\left\{\operatorname{diam}\left(\phi_{\omega}(X)\right)\right\}=0$. Hence, the map $\pi: I^{\infty} \rightarrow X$, given by $\pi(\omega)=\bigcap_{n \geq 0} \phi_{\left.\omega\right|_{n}}(X)$, is uniformly continuous. Now, the limit set $J=J_{S}$ of the system $S$ can be defined as the range of the map $\pi$, that is, we define

$$
J=\pi\left(I^{\infty}\right)
$$

In order to introduce the notion of tameness we define, for every $i \in \Omega$,

$$
X_{i}=\bigcup_{j \in I \backslash\{i\}} \phi_{j}(X) .
$$

We call a parabolic conformal iterated function system $S=\left\{\phi_{i}: i \in I\right\}$ tame if $x_{i} \notin X_{i}$, for every $i \in \Omega$ and $x_{i} \neq x_{j}$ if $i \neq j$. Also, we say that $S$ satisfies the Super Strong Open Set Condition (SSOSC) if the following condition is satisfied:

$$
\partial X \cap \bigcup_{i \in I} \phi_{i}(X)=\left\{x_{i}: i \in \Omega\right\}
$$

Unless stated otherwise, for the remaining part of this section we shall assume that $S$ is a tame parabolic finite conformal iterate function system satisfying (SSOSC). The tameness of the system $S$ and formula (2.7) imply

$$
B\left(\bigcup_{i \in I \backslash \Omega} \phi_{i}(X), 2 \hat{R}\right) \subset \operatorname{Int} X .
$$

Also, for each $\omega \in I^{*}$ and every $A \subset B\left(x_{i}, 2 \hat{R}\right)$ we have that

$$
\phi_{\omega}(A) \cap J=\phi_{\omega}(A \cap J) .
$$

Note that in order to derive the latter formula, we have to use the fact that the system $S$ is tame. Furthermore, for all $i \in \Omega, \omega \in I^{*}$ we have

$$
\pi^{-1}\left(\pi\left(\omega i^{\infty}\right)\right)=\omega i^{\infty} .
$$

Following [MU1], given $t \geq 0$, a Borel probability measure $m$ is called $t$-conformal for the system $S$ if $m(J)=1$ and if for every Borel set $A \subset X$ and for each $i, j \in I$ with $i \neq j$, we have

$$
m\left(\phi_{i}(A)\right)=\int_{A}\left|\phi_{i}^{\prime}\right|^{t} d m
$$

and

$$
m\left(\phi_{i}(X) \cap \phi_{j}(X)\right)=0 .
$$

Recall that a parabolic system $S$ is called regular if and only if there exists a $t$-conformal measure (cf. [MU1]). Then $t=h$ is the Hausdorff dimension of the limit set (see [MU1]). Combining Theorem 1.4 in [MU2] and Corollary 5.8 in [MU1], we immediately have the following result:

Theorem 2.1. A parabolic finite iterated function system is regular. 
Hence, since the systems we consider in this paper are finite, it follows that they are regular. The associated $h$-conformal measure will always be denoted by $m$. We shall require the following distortion properties:

Lemma 2.2. There exists a positive constant $R^{*}<\hat{R}$ such that the following holds: for each hyperbolic word $\tau \in I^{*}$ and for every $\omega \in I^{\infty}$ we have that $\phi_{\tau}$ is well-defined on $B\left(\pi(\omega), R^{*}\right)$. Moreover

$$
\frac{\left|\phi_{\tau}^{\prime}(y)\right|}{\left|\phi_{\tau}^{\prime}(x)\right|} \leq K \quad \text { for all } x, y \in B\left(\pi(\omega), R^{*}\right)
$$

and

$$
\begin{aligned}
K^{-h}\left|\phi_{\tau}^{\prime}(\pi(\omega))\right|^{h} m(B(\pi(\omega), r)) & \leq m\left(\phi_{\tau}(B(\pi(\omega), r))\right) \\
& \leq K^{h}\left|\phi_{\tau}^{\prime}(\pi(\omega))\right|^{h} m(B(\pi(\omega), r))
\end{aligned}
$$

for every $r \in\left[0, R^{*}\right]$.

Proof. The statement that $\phi_{\tau}: B\left(\pi(\omega), R^{*}\right) \rightarrow \mathbb{R}^{d}$ is well-defined and the first distortion property of the lemma are immediate consequences of the fact that $R^{*}<\hat{R}<\operatorname{Dist}(X, \partial V)$ and property (7) at the beginning of this section. In order to derive the second distortion property of the lemma, choose $0<R^{*}<\hat{R}$ sufficiently small such that, for each $i \in \Omega$,

$$
B\left(\phi_{i}(X) \cap\left(\mathbb{R}^{d} \backslash B\left(x_{i}, \hat{R}\right)\right), 2 R^{*}\right) \subset \operatorname{Int} X .
$$

If $\pi(\omega) \in \phi_{i}(X)$ for some $i \in \Omega$, and if $\left\|\pi(\omega)-x_{i}\right\| \geq \hat{R}$, then $B\left(\pi(\omega), 2 R^{*}\right) \subset$ Int $X$. The proof in this case then follows immediately from a combination of the conformality of the measure $m$ and distortion property (7). In the case that $\pi(\omega) \in \phi_{i}(X) \cap B\left(x_{i}, \hat{R}\right)$, it follows that $B\left(\pi(\omega), R^{*}\right) \subset B\left(x_{i}, 2 \hat{R}\right)$. Using (2.9) and the conformality of $m$, we obtain

$$
\begin{aligned}
m\left(\phi_{\tau}((B(\pi(\omega), r)))\right. & =m\left(\phi_{\tau}(B(\pi(\omega), r)) \cap J\right) \\
& =m\left(\phi_{\tau}(B(\pi(\omega), r) \cap J)\right) \\
& =\int_{B(\pi(\omega), r) \cap J}\left|\phi_{\tau}^{\prime}\right|^{h} d m=\int_{B(\pi(\omega), r)}\left|\phi_{\tau}^{\prime}\right|^{h} d m,
\end{aligned}
$$

and hence the first distortion property of the lemma gives the proof in this case. Finally, if $\pi(\omega) \notin \bigcup_{i \in \Omega} \phi_{i}(X)$, then $\pi(\omega) \in \phi_{j}(X)$ for some $j \in I \backslash \Omega$. In this case (2.8) implies that $B\left(\pi(\omega), 2 R^{*}\right) \subset \operatorname{Int} X$, and hence the statement of the lemma follows immediately from (7) and the conformality of $m$. This proves the lemma.

The following fact easily follows from the local analysis of parabolic points done in [MU2]: 
Lemma 2.3. Assuming that $R^{*}>0$ is sufficiently small, there exists a constant $C_{1}>0$ such that for every $i \in \Omega$ and every $r \in\left(0, R^{*}\right]$, the intersection $J \cap B\left(x_{i}, r\right) \backslash\left\{x_{i}\right\}$ is contained in a central cone contained in Int $X$ with vertex $x_{i}$ and an angle $\leq C_{1} r^{p_{i}}$.

As an immediate consequence of this lemma and (2.9) we get the following:

Lemma 2.4. There exists a constant $C_{1}>0$ such that for every $i \in \Omega$, every $r \in\left(0, R^{*}\right]$, and every hyperbolic word $\omega$ the intersection

$$
J \cap \bar{B}\left(\phi_{\omega}\left(x_{i}\right), r\left|\phi_{\omega}^{\prime}\left(x_{i}\right)\right|\right)
$$

is contained in a central cone with vertex $x_{i}$ and an angle $\leq C_{2} r^{p_{i}}$.

We are now in a position to prove the following distortion property:

Lemma 2.5. There exist constants $\rho, R_{*}>0$ such that for every $i \in \Omega$, $x \in J \cap B\left(x_{i}, R_{*}\right)$, and for each $\omega \in I^{*}$ the map $\phi_{\omega}$ is well-defined on $B\left(x, \rho\left\|x-x_{i}\right\|\right)$ and

$$
\frac{\left|\phi_{\omega}^{\prime}(z)\right|}{\left|\phi_{\omega}^{\prime}(y)\right|} \leq K \quad \text { for all } y, z \in B\left(x, \rho\left\|x-x_{i}\right\|\right),
$$

and furthermore, for every positive $r \leq \rho\left\|x-x_{i}\right\|$ we have

$$
K^{-h}\left|\phi_{\omega}^{\prime}(x)\right|^{h} m(B(x, r)) \leq m\left(\phi_{\omega}(B(x, r))\right) \leq K^{h}\left|\phi_{\omega}^{\prime}(x)\right|^{h} m(B(x, r)) .
$$

Proof. In view of Lemma 2.3 there exists $R_{*}>0$ and $\rho \in(0,1 / 2)$ such that $B\left(x, 2 \rho\left\|x-x_{i}\right\|\right) \subset \operatorname{Int} X$ for all $x \in J \cap B\left(x_{i}, R_{*}\right)$. Therefore, all the maps $\phi_{\omega}: B\left(x, 2 \rho\left\|x-x_{i}\right\|\right) \rightarrow$ Int $X$ are well-defined, and the second part of our lemma follows from the first part. The first part in turn in the case when $d=1$ is contained in Lemma 2.6 of [U2]. In the case $d=2$ it follows immediately from Koebe's distortion theorem and the observation that the complex conjugation is an isometry. In the case $d \geq 3$ it follows from the inequality following formula (4.9) in the proof of Theorem 4.13 in [MU2] that, with $Y=\overline{B\left(x, \rho\left\|x-x_{i}\right\|\right)}, W=B\left(x, 2 \rho\left\|x-x_{i}\right\|\right)$, one gets $\left|\phi_{\omega}^{\prime}(z)\right| /\left|\phi_{\omega}^{\prime}(y)\right| \leq 4$. We are done.

The constants $R_{*}$ and $R^{*}$ of Lemma 2.2 and Lemma 2.5 will be crucial in the sequel. For later use we define

$$
R:=\min \left\{R_{*}, R^{*}\right\} .
$$

\section{The geometry of conformal measures}

The main result in this section is the derivation of a 'global formula' for the conformal measure associated with a tame parabolic finite iterated function system. This formula describes in a uniform way the scaling of this measure at arbitrary points in the associated limit set. Our elaboration of this formula follows closely the discussion in $[\mathbf{S V}]$ and $[\mathbf{S U 2}]$, where we obtained 
this type of formula for geometrically finite Kleinian groups with parabolic elements and for parabolic rational maps.

The section is split into two subsections. In the first we give an estimate for the conformal measure around parabolic points. In the second we then derive the global formula. Subsequently, as a first application of this formula, we obtain a first rough description of how the conformal measure relates to the geometric concepts Hausdorff measure and packing measure.

3.1. The conformal measure around parabolic points. We begin this subsection by recalling the following estimates for tame parabolic systems. For $d \geq 2$ a proof can be found in [MU2] (Section 4). For $d=1$ the estimates are obtained immediately from the considerations in [U2].

Proposition 3.1. Let $S$ be a tame parabolic system. Then there exists a constant $Q \geq 1$ and an integer $q \geq 0$ such that for every parabolic index $i \in I$ there exists an integer $p_{i} \geq 1$ such that for every $j \in I \backslash\{i\}$ and for all $n, k \geq 1$ we have

$$
\begin{gathered}
Q^{-1} n^{-\frac{p_{i}+1}{p_{i}}} \leq \inf _{X}\left\{\left|\phi_{i^{n} j}^{\prime}\right|\right\},\left\|\phi_{i^{n} j}^{\prime}\right\|, \operatorname{diam}\left(\phi_{i^{n} j}(X)\right) \leq Q n^{-\frac{p_{i}+1}{p_{i}}}, \\
Q^{-1} n^{-\frac{1}{p_{i}}} \leq \operatorname{Dist}\left(x_{i}, \phi_{i^{n}}\left(X_{i}\right)\right) \leq \operatorname{Dist}\left(x_{i}, \phi_{i^{n}}\left(X_{i}\right)\right) \leq Q n^{-\frac{1}{p_{i}}}, \\
\operatorname{Dist}\left(\phi_{i^{n}}\left(X_{i}\right), \phi_{i^{k}}\left(X_{i}\right)\right) \leq Q\left|n^{-\frac{1}{p_{i}}}-k^{-\frac{1}{p_{i}}}\right| .
\end{gathered}
$$

Furthermore, for $|n-k| \geq q$ we have

$$
\operatorname{Dist}\left(\phi_{i^{n}}\left(X_{i}\right), \phi_{i^{k}}\left(X_{i}\right)\right) \geq Q\left|n^{-\frac{1}{p_{i}}}-k^{-\frac{1}{p_{i}}}\right| .
$$

The following lemma gives the main result of this section:

Lemma 3.2. Let $m$ denote the $h$-conformal measure of the finite parabolic system $S$. For each $\kappa>0$ there exists $C_{\kappa}>0$ such that for every parabolic index $i$ and for every $x \in \bar{J}$ we have

$$
C_{\kappa}^{-1}\left\|x-x_{i}\right\|^{h+(h-1) p_{i}} \leq m\left(B\left(x, \kappa\left\|x-x_{i}\right\|\right)\right) \leq C_{\kappa}\left\|x-x_{i}\right\|^{h+(h-1) p_{i}} .
$$

In particular, the constant $C_{\kappa}$ depends continuously on $\kappa$.

Proof. Since the support of $m$ is equal to $\bar{J}$, we may assume without loss of generality that $\left\|x-x_{i}\right\| \leq \Delta$ for some fixed $0<\Delta \leq R$. Let $x=\pi(\omega)$ and $\omega \in I^{\infty}$ be given. Then $\omega=i^{n} j \tau$, where $j \neq i, n \geq 1$, and $\tau \in I^{\infty}$. Assuming $\Delta$ to be chosen sufficiently small, (3.1) implies that

$$
n \geq 2 Q^{2} \kappa^{-1} .
$$

For the proof of the first inequality in the measure estimate of the lemma, let

$$
T:=\left\{k: \operatorname{Dist}\left(\phi_{i^{k} j}(X), \phi_{i^{n} j}(X)\right) \leq \kappa\left\|x-x_{i}\right\|-\operatorname{diam}\left(\phi_{i^{n} j}(X)\right)\right\} .
$$


Using (3.1), we deduce that

$$
m\left(B\left(x, \kappa\left\|x-x_{i}\right\|\right)\right) \geq \sum_{k \in T} m\left(\phi_{i^{k} j}(X)\right) \sum_{k \in T} \inf \left\{\left|\phi_{i^{k} j}^{\prime}\right|\right\}^{h} \geq \sum_{k \in T} Q^{-h} k^{-\frac{p_{i}+1}{p_{i}} h} .
$$

Using (3.2) and (3.1), we have that if

$$
Q\left|n^{-\frac{1}{p_{i}}}-k^{-\frac{1}{p_{i}}}\right| \leq \kappa Q^{-1} n^{-\frac{1}{p_{i}}}-Q n^{-\frac{p_{i}+1}{p_{i}}}
$$

then it follows that $k \in T$. Hence in particular, if $k \geq n$ and if

$$
Q\left(n^{-\frac{1}{p_{i}}}-k^{-\frac{1}{p_{i}}}\right) \leq \kappa Q^{-1} n^{-\frac{1}{p_{i}}}-Q n^{-\frac{p_{i}+1}{p_{i}}},
$$

then $k \in T$. Clearly, the statement in (3.6) is equivalent to

$$
Q k^{-\frac{1}{p_{i}}} \geq\left(Q-\kappa Q^{-1}\right) n^{-\frac{1}{p_{i}}}+Q n^{-\frac{p_{i}+1}{p_{i}}}
$$

Also, (3.5) implies that $Q n^{-\frac{p_{i}+1}{p_{i}}} \leq \kappa(2 Q)^{-1} n^{-\frac{1}{p_{i}}}$. Therefore, if $k \geq n$ and $Q k^{-\frac{1}{p_{i}}} \geq\left(Q-\kappa Q^{-1}\right) n^{-\frac{1}{p_{i}}}+\kappa(2 Q)^{-1} n^{-\frac{1}{p_{i}}}$, or equivalently if $k \geq n$ and $k \leq\left(1-\frac{\kappa}{2 Q}\right)^{-\frac{1}{p_{i}}}$, then $k \in T$. It now follows that there exists a constant $\widetilde{C}_{\kappa}>0$ (which depends continuously on $\kappa$ ) such that

$$
\begin{aligned}
m\left(B\left(x, \kappa\left\|x-x_{i}\right\|\right)\right) & \geq Q^{-h} \sum_{k=n}^{\left(1-\frac{\kappa}{2 Q}\right)^{-\frac{1}{p_{i}}}} k^{-\frac{p_{i}+1}{p_{i}} h} \\
& \geq Q^{-h}\left(1-\frac{p_{i}+1}{p_{i}} h\right)\left(\left(1-\frac{\kappa}{2 Q}\right)^{-\frac{1}{p_{i}}\left(1-\frac{p_{i}+1}{p_{i}} h\right)}-1\right) n^{-\frac{p_{i}+1}{p_{i}} h} \\
& \geq \widetilde{C}_{\kappa} n^{-\frac{h+(h-1) p_{i}}{p_{i}}} .
\end{aligned}
$$

Hence, since by (3.6) we have $\left\|x-x_{i}\right\| \leq Q n^{-\frac{1}{p_{i}}}$, it follows that

$$
m\left(B\left(x, \kappa\left\|x-x_{i}\right\|\right)\right) \geq \widetilde{C}_{\kappa} Q^{h+(h-1) p_{i}}\left\|x-x_{i}\right\|^{h+(h-1) p_{i}} .
$$

In order to prove the second inequality in the measure estimate of the lemma, note that $Q k^{-\frac{1}{p_{i}}} \leq(1+\kappa)\left\|x-x_{i}\right\|$ if and only if $k \geq\left(Q^{-1}(1+\kappa)\left\|x-x_{i}\right\|\right)^{-p_{i}}$. 
Using this observation, (3.2) and (3.1), we obtain

$$
\begin{aligned}
m\left(B\left(x, \kappa\left\|x-x_{i}\right\|\right)\right) & \leq m\left(B\left(x_{i},(1+\kappa)\left\|x-x_{i}\right\|\right)\right. \\
& \leq \sum_{j \neq i} \sum_{k=\left(Q^{-1}(1+\kappa)\left\|x-x_{i}\right\|\right)^{-p_{i}}} m\left(\phi_{i^{k} j}(X)\right) \\
& \leq \sum_{j \neq i}\left\|\phi_{k=\left(Q^{-1}(1+\kappa)\left\|x-x_{i}\right\|\right)^{-p_{i}}}\right\|_{i^{h}} \sum \sum_{j \neq i} \sum_{k=\left(Q^{-1}(1+\kappa)\left\|x-x_{i}\right\|\right)^{-p_{i}}} \sum^{-\frac{p_{i}+1}{p_{i}} h} \\
& \leq 2 Q\left(k^{h \frac{p_{i}+1}{p_{i}}-1}\right)^{-1} \\
& \leq\left(Q^{-1}(1+\kappa)\left\|x-x_{i}\right\|\right)^{-p_{i}\left(1-\frac{p_{i}+1}{p_{i}} h\right)} \\
& =\hat{C}_{\kappa}\left\|x-x_{i}\right\|^{h+(h-1) p_{i}},
\end{aligned}
$$

where $\hat{C}_{\kappa}<\infty$ denotes a positive constant depending continuously on $\kappa$.

Corollary 3.3. There exists a constant $C \geq 1$ such that for each $i \in \Omega$ and for all $0<r \leq 2 \operatorname{diam}(X)$ we have

$$
C^{-1} r^{h+(h-1) p_{i}} \leq m\left(B\left(x_{i}, r\right)\right) \leq C r^{h+(h-1) p_{i}} .
$$

Proof. Let $j \neq i$, and choose $n \geq 1$ to be the least integer such that $Q^{-1} n^{-\frac{1}{p_{i}}} \leq r$. Let $x \in \phi_{i^{n-1} j}(X)$ be fixed. By (3.2) and Lemma 3.2, we have

$$
\begin{aligned}
m\left(B\left(x_{i}, r\right)\right) & \leq m\left(x, 2\left\|x-x_{i}\right\|\right) \\
& \leq C_{2}\left\|x-x_{i}\right\|^{h+(h-1) p_{i}} \leq C_{2} Q(n-1)^{-\frac{h+(h-1) p_{i}}{p_{i}}} \\
& \ll n^{-\frac{h+(h-1) p_{i}}{p_{i}}} \ll\left(\frac{Q}{2}\right)^{h+(h-1) p_{i}} r^{h+(h-1) p_{i}} .
\end{aligned}
$$

Now, let $k \geq 1$ denote the least integer such that $Q k^{-\frac{1}{p_{i}}} \leq r / 2$, and let $y \in \phi_{i^{k} j}(X)$ be fixed. Similar as above, (3.2) and Lemma 3.2 imply that

$$
\begin{aligned}
m\left(B\left(x_{i}, r\right)\right) & \geq m\left(B\left(y, \| y-x_{i} \mid\right)\right) \\
& \geq C_{1}\left\|y-x_{i}\right\|^{h+(h-1) p_{i}} \geq C_{1} Q^{-\left(h+(h-1) p_{i}\right)} k^{-\frac{h+(h-1) p_{i}}{p_{i}}} \\
& \gg(k-1)^{-\frac{h+(h-1) p_{i}}{p_{i}}} \geq 2^{-\left(h+(h-1) p_{i}\right)} r^{h+(h-1) p_{i}} .
\end{aligned}
$$

Lemma 3.4. For every $\kappa>0$ there exists $D_{\kappa} \geq 1$ such that for each $i \in \Omega$, for every sufficiently small $r>0$, and for all $x \in J \cap B\left(x_{i}, \kappa^{-1} r\right)$ we have

$$
D_{\kappa}^{-1} r^{h+(h-1) p_{i}} \leq m(B(x, r)) \leq D_{\kappa} r^{h+(h-1) p_{i}} .
$$


Proof. Since $B(x, r) \subset B\left(x_{i},\left\|x-x_{i}\right\|+r\right) \subset B\left(x_{i},\left(1+\kappa^{-1}\right) r\right)$, it follows from Corollary 3.3 that

$$
m(B(x, r)) \leq C\left(1+\kappa^{-1}\right)^{h+(h-1) p_{i}} r^{h+(h-1) p_{i}} .
$$

Now, if $r \leq 2\left\|x-x_{i}\right\|$, then $r=\alpha\left\|x-x_{i}\right\|$ for some $\alpha$ such that $\kappa \leq \alpha \leq 2$. By Lemma 3.2, we have $C_{\alpha} \leq \bar{C}:=\sup \left\{C_{t}: t \in[\kappa, 2]\right\}<\infty$. Hence, using Lemma 3.2 once again, it follows that

$$
\begin{aligned}
m(B(x, r)) & =m\left(B\left(x, \alpha\left\|x-x_{i}\right\|\right) \geq C_{\alpha}^{-1}\left\|x-x_{i}\right\|^{h+(h-1) p_{i}}\right. \\
& \geq \bar{C}^{-1}\left(\frac{r}{\alpha}\right)^{h+(h-1) p_{i}} \\
& \geq \bar{C} \max \left\{\kappa^{-\left(h+(h-1) p_{i}\right)}, 2^{-\left(h+(h-1) p_{i}\right)}\right\} r^{h+(h-1) p_{i}} .
\end{aligned}
$$

Otherwise if $r \geq 2\left\|x-x_{i}\right\|$, then Corollary 3.3 implies

$$
m(B(x, r)) \geq m\left(B\left(x_{i}, r / 2\right)\right) \geq C\left(\frac{r}{2}\right)^{h+(h-1) p_{i}}=C 2^{-\left(h+(h-1) p_{i}\right)} r^{h+(h-1) p_{i}} .
$$

Combining this last estimate with (3.8) and (3.9), the lemma follows.

3.2. The global formula for the conformal measure. An element $\omega \in$ $I^{\infty}$ is called preparabolic if and only if $\sigma^{k} \omega=i^{\infty}$ for some $k \geq 0$ and some $i \in \Omega$. The set of all preparabolic elements will be denoted by $I_{p}^{\infty}$. Also, a limit point that is not a preparabolic element will be referred to as radial, and we write $I_{r}^{\infty}$ to denote the set of all radial points.

For each $\omega \in I^{\infty}$ we fix an increasing sequence of integers $\left\{n_{j}(\omega)\right\}_{j=1}^{k(\omega)}$ as follows: assume that $n_{j}(\omega)$ is defined, then we define $n_{j+1}(\omega)$ to be the smallest index greater than $n_{j}(\omega)$ such that either $\omega_{n_{j+1}(\omega)}$ is hyperbolic or $\omega_{n_{j+1}(\omega)} \neq \omega_{n_{j+1}(\omega)-1}$ (note that $n_{1}(\omega)$ is well-defined). In case $n_{j+1}(\omega)$ does not exist, then $j=k(\omega)$. Note that if $n_{j+1}(\omega) \geq n_{j}(\omega)+2$, then there exists a unique parabolic index $i=i(\omega, j)$ such that $\omega_{l}=i$ for all $n_{j}(\omega) \leq l \leq n_{j+1}(\omega)$. Furthermore, if $n_{j+1}(\omega)=n_{j}(\omega)+1$, then $i(\omega, j)$ denotes some arbitrary element of $\Omega$. Observe that $k(\omega)=\infty$ if and only if $\omega \in I_{r}^{\infty}$. For each $j$, we define

$$
r_{j}(\omega):=R\left|\phi_{\left.\omega\right|_{n_{j}(\omega)}}^{\prime}\left(\pi\left(\sigma^{n_{j}(\omega)} \omega\right)\right)\right|,
$$

and we refer to the sequence $\left\{r_{j}(\omega)\right\}_{j=1}^{k(\omega)}$ as the hyperbolic zoom of $\omega$. Note that by the chain rule and by property (6) of Section $2,\left\{r_{j}(\omega)\right\}_{j=1}^{k(\omega)}$ is a strictly decreasing sequence. Hence, for each $\omega \in I_{r}^{\infty}$ and every positive

$$
r \leq \widetilde{R}=\min \left\{\inf \left\{\left|\phi_{i}^{\prime}\right|: i \notin \Omega\right\}, \inf \left\{\left|\phi_{i j}^{\prime}\right|: i \in \Omega, j \neq i\right\}\right\},
$$

there exists a unique $j \geq 1$ such that $r_{j+1}(\omega)<r \leq r_{j}(\omega)$. For a given $\omega$ and $r$, the neighbours $r_{j+1}(\omega)$ and $r_{j}(\omega)$ thus determined in the hyperbolic zoom of $\omega$ will be denoted by $r_{*}(\omega)$ and $r^{*}(\omega)$ respectively. Also, in this situation 
we shall write $i(\omega, r)$ to denote the parabolic element $i(\omega, j)$. Finally we define the function $\zeta$, given for $\omega \in I^{\infty}$ and $r>0$ by

$$
\zeta(\omega, r):=\frac{m(B(x, r))}{r^{h}} .
$$

The following theorem is the main result of this section:

Theorem 3.5 (Global formula for conformal measures). Let $S$ be a tame parabolic finite iterated function system satisfying the (SSOSC). Then, for each $\omega \in I_{r}^{\infty}$ and every $0<r \leq \widetilde{R}$, and setting $i=i(\omega, r)$, we have

$$
\zeta(\omega, r) \asymp \begin{cases}\left(\frac{r}{r^{*}(\omega)}\right)^{(h-1) p_{i}} & \text { for } r^{*}(\omega) \geq r \geq r^{*}(\omega)\left(\frac{r_{*}(\omega)}{r^{*}(\omega)}\right)^{\frac{1}{p_{i}+1}} \\ \left(\frac{r_{*}(\omega)}{r}\right)^{h-1} & \text { for } r_{*}(\omega) \leq r \leq r^{*}(\omega)\left(\frac{r_{*}(\omega)}{r^{*}(\omega)}\right)^{\frac{1}{p_{i}+1}} .\end{cases}
$$

Proof. Let $\omega \in I_{r}^{\infty}$ and $0<r \leq \widetilde{R}$ be fixed. For ease of notation, throughout the proof we shall suppress the dependence on $\omega$ in some of the appearing quantities. Let $j$ be determined by the condition $r^{*}=r_{j}$. Hence, $r_{*}=r_{j+1}$. By (3.2), we have

$$
\left\|\pi\left(\sigma^{n_{j}} \omega\right)-x_{i}\right\|=\left\|\pi\left(\phi_{i^{n_{j+1}-n_{j}-1} \omega_{n_{j+1}}}\left(\pi\left(\sigma^{n_{j+1}} \omega\right)\right)\right)-x_{i}\right\| \asymp\left(n_{j+1}-n_{j}\right)^{-\frac{1}{p_{i}}} .
$$

Using the chain rule and (3.1), we obtain

$$
\begin{aligned}
1 & =r_{j+1}\left|\phi_{\left.\omega\right|_{j}}^{\prime}\left(\pi\left(\sigma^{n_{j}} \omega\right)\right)\right|^{-1}\left|\phi_{\left.\sigma^{n_{j}}\right|_{n_{j+1}-n_{j}-1}}^{\prime}\left(\pi\left(\sigma^{n_{j+1}} \omega\right)\right)\right|^{-1} \\
& \asymp\left(\frac{r_{j+1}}{r_{j}}\right)\left(n_{j+1}-n_{j}\right)^{\frac{p_{i}+1}{p_{i}}}=\left(\frac{r_{*}}{r^{*}}\right)\left(n_{j+1}-n_{j}\right)^{\frac{p_{i}+1}{p_{i}}} .
\end{aligned}
$$

Hence,

$$
\left(\frac{r_{*}}{r^{*}}\right)^{\frac{1}{p_{i}}} \asymp\left\|\pi\left(\sigma^{n_{j}} \omega\right)-x_{i}\right\|
$$

This implies that if

$$
r \geq r^{*}(\omega)\left(\frac{r_{*}(\omega)}{r^{*}(\omega)}\right)^{\frac{1}{p_{i}+1}}
$$

then

$$
\left|\phi_{\left.\omega\right|_{n_{j}}}^{\prime}\left(\pi\left(\sigma^{n_{j}} \omega\right)\right)\right| \cdot\left\|\pi\left(\sigma^{n_{j}} \omega\right)-x_{i}\right\| \leq r \leq\left|\phi_{\left.\omega\right|_{n_{j}}}^{\prime}\left(\pi\left(\sigma^{n_{j}} \omega\right)\right)\right|,
$$

and hence

$$
\left\|\pi\left(\sigma^{n_{j}} \omega\right)-x_{i}\right\| \leq \frac{r}{\left|\phi_{\left.\omega\right|_{n_{j}}}^{\prime}\left(\pi\left(\sigma^{n_{j}} \omega\right)\right)\right|} \leq 1
$$


Now, using property (7) (note that $\phi_{\left.\omega\right|_{n_{j}}}$ is hyperbolic) and Lemma 3.4, it follows that

$$
\begin{aligned}
m(B(\pi(\omega), r)) & \asymp\left|\phi_{\left.\omega\right|_{n_{j}}}^{\prime}\left(\pi\left(\sigma^{n_{j}} \omega\right)\right)\right|^{h} m\left(B\left(\pi\left(\sigma^{n_{j}} \omega\right), r\left|\phi_{\left.\omega\right|_{n_{j}}}^{\prime}\left(\pi\left(\sigma^{n_{j}} \omega\right)\right)\right|^{-1}\right)\right) \\
& \asymp r_{j}^{h}\left(r r_{j}^{-1}\right)^{h+(h-1) p_{i}}=r^{h}\left(\frac{r}{r_{j}}\right)^{(h-1) p_{i}}=r^{h}\left(\frac{r}{r^{*}}\right)^{(h-1) p_{i}} .
\end{aligned}
$$

This proves the first case in the theorem. We are now left to consider the case in which

$$
r \leq r^{*}(\omega)\left(\frac{r_{*}(\omega)}{r^{*}(\omega)}\right)^{\frac{1}{p_{i}+1}} .
$$

Because of (3.10) (after arranging for appropriate constants), this means that

$$
\left|\phi_{\left.\omega\right|_{n_{j+1}}}^{\prime}\left(\pi\left(\sigma^{n_{j+1}} \omega\right)\right)\right| \leq r \leq \rho\left|\phi_{\omega_{n_{j}}}^{\prime}\left(\pi\left(\sigma^{n_{j}} \omega\right)\right)\right| \cdot\left\|\pi\left(\sigma^{n_{j}} \omega\right)-x_{i}\right\|,
$$

where $0<\rho<1$ is the constant obtained in Lemma 2.5. Therefore, there exists $n_{j} \leq u \leq n_{j+1}-1$ such that

$\rho\left|\phi_{\left.\omega\right|_{u+1}}^{\prime}\left(\pi\left(\sigma^{u+1} \omega\right)\right)\right| \cdot\left\|\pi\left(\sigma^{u+1} \omega\right)-x_{i}\right\| \leq r \leq \rho\left|\phi_{\left.\omega\right|_{u}}^{\prime}\left(\pi\left(\sigma^{u} \omega\right)\right)\right| \cdot\left\|\pi\left(\sigma^{u} \omega\right)-x_{i}\right\|$.

In particular, this implies that

$$
r \asymp \rho\left|\phi_{\left.\omega\right|_{u}}^{\prime}\left(\pi\left(\sigma^{u} \omega\right)\right)\right| \cdot\left\|\pi\left(\sigma^{u} \omega\right)-x_{i}\right\| .
$$

Thus, by using the conformality of $m$, Lemma 2.5 and Lemma 3.2, it follows that

$$
\begin{aligned}
m(B(\pi(\omega), r)) & \asymp\left|\phi_{\left.\omega\right|_{u}}^{\prime}\left(\pi\left(\sigma^{u} \omega\right)\right)\right|^{h} m\left(B\left(\pi\left(\sigma^{u} \omega\right), \rho\left\|\pi\left(\sigma^{u} \omega\right)-x_{i}\right\|\right)\right) \\
& \asymp\left|\phi_{\left.\omega\right|_{u}}^{\prime}\left(\pi\left(\sigma^{u} \omega\right)\right)\right|^{h}\left\|\pi\left(\sigma^{u} \omega\right)-x_{i}\right\|^{h+(h-1) p_{i}} \\
& \asymp r^{h}\left\|\pi\left(\sigma^{u} \omega\right)-x_{i}\right\|^{(h-1) p_{i}} .
\end{aligned}
$$

On the other hand, the chain rule, (3.1) and (3.2) imply that

$$
\begin{aligned}
1 & =r_{j+1}\left|\phi_{\left.\omega\right|_{u}}^{\prime}\left(\pi\left(\sigma^{u} \omega\right)\right)\right|^{-1}\left|\phi_{\left.\sigma^{u} \omega\right|_{n_{j+1}-u-1}}^{\prime}\left(\pi\left(\sigma^{n_{j+1}} \omega\right)\right)\right|^{-1} \\
& \asymp r_{*}\left|\phi_{\omega \mid u}^{\prime}\left(\pi\left(\sigma^{u} \omega\right)\right)\right|^{-1}\left(n_{j+1}-u\right)^{\frac{p_{i}+1}{p_{i}}}
\end{aligned}
$$

as well as

$$
\left\|\pi\left(\sigma^{u} \omega\right)-x_{i}\right\|^{-\left(p_{i}+1\right)} \asymp\left(n_{j+1}-u\right)^{\frac{p_{i}+1}{p_{i}}} .
$$

These two latter comparabilities together with (3.11) show that

$$
r \asymp r_{*}\left\|\pi\left(\sigma^{u} \omega\right)-x_{i}\right\|^{-\left(p_{i}+1\right)}\left\|\pi\left(\sigma^{u} \omega\right)-x_{i}\right\|=r_{*}\left\|\pi\left(\sigma^{u} \omega\right)-x_{i}\right\|^{-p_{i}} .
$$

Hence, $\left\|\pi\left(\sigma^{u} \omega\right)-x_{i}\right\| \asymp\left(r_{*} / r\right)^{1 / p_{i}}$, which together with (3.12) implies that

$$
m(B(\pi(\omega), r)) \asymp r^{h}\left(\frac{r_{*}}{r}\right)^{h-1} .
$$

This proves the second case in the theorem. 
The following corollaries are immediate consequences of the previous theorem.

Corollary 3.6. If $\omega \in I_{r}^{\infty}$, then for each $j \geq 1$ we have

$$
m\left(B\left(\pi(\omega), r_{j}(\omega)\right)\right) \asymp r_{j}(\omega)^{h} .
$$

Corollary 3.7. The conformal measure $m$ is a doubling measure. This means that for every $c>0$ there exists $B>0$ such that for each $z \in J$ and every $r>0$ we have

$$
m(B(z, c r)) \leq B m(B(z, r)) .
$$

Finally, as a first nontrivial application of Theorem 3.5 we derive an alternative proof of the following geometrical fact, which was obtained under slightly weaker assumptions in $[\mathbf{M U} 2]$. For this let $\mathrm{H}^{t}$ and $\mathrm{P}^{t}$ denote the $t$-dimensional Hausdorff and packing measure respectively.

Theorem 3.8. If $S$ is a tame finite parabolic system satisfying the (SSOSC), then the following hold:

(a) If $h>1$, then $0<\mathrm{H}^{h}(J)<\infty$ and $\mathrm{P}^{h}(J)=\infty$.

(b) If $h=1$, then $0<\mathrm{H}^{h}(J), \mathrm{P}^{h}(J)<\infty$.

(c) If $h<1$, then $0<\mathrm{P}^{h}(J)<\infty$ and $\mathrm{H}^{h}(J)=0$.

Additionally, if either measure $\mathrm{H}^{h}$ or $\mathrm{P}^{h}$ is finite and positive, then its normalized version is equal to the conformal measure $m$.

Proof. In [MU1] (Lemma 5.6 and Theorem 5.7) it was shown that for a tame finite parabolic system satisfying the (SSOSC) the $h$-conformal measure $m$ is atomless. This combined with Corollary 3.6 and the inverse Frostmann lemma (see [MU3]) implies that $\mathrm{H}^{h}(J)<\infty$ and $\mathrm{P}^{h}(J)>0$. Now, if $h \geq 1$, then Theorem 3.5 immediately gives that, for every $x \in \pi\left(I_{r}^{\infty}\right)$,

$$
\limsup _{r \rightarrow 0} \frac{m(B(x, r))}{r^{h}} \ll 1,
$$

which implies that $\mathrm{H}^{h}(J)>0$. If in addition $x=\pi(\omega)$, for $\omega \in I_{r}^{\infty}$ containing arbitrarily long blocks of $i$ 's for some $i \in \Omega$, then

$$
\begin{aligned}
\liminf _{r \rightarrow 0} \frac{m(B(x, r))}{r^{h}} & \leq \liminf _{r \rightarrow 0} \zeta\left(\omega, \rho r^{*}(\omega)\left(\frac{r_{*}(\omega)}{r^{*}(\omega)}\right)^{\frac{1}{p_{i}+1}}\right) \\
& =\liminf _{r \rightarrow 0}\left(\frac{r_{*}(\omega)}{r^{*}(\omega)}\right)^{\frac{(h-1) p_{i}}{p_{i}+1}}=0 .
\end{aligned}
$$

Now, by ergodicity of the measure $m$ (see [MU2], Corollary 5.11) and since $m$ is positive on open sets, it follows that $m$-almost everywhere we have

$$
\liminf _{r \rightarrow 0} \frac{m(B(x, r))}{r^{h}}=0 .
$$


We conclude that $\mathrm{P}^{h}(J)=\infty$, which proves case (a) of the theorem. Case (b) is an immediate consequence of Theorem 3.5. The proof of case (c) is analogous to the proof of case (a), and we omit it.

\section{Metrical Diophantine analysis}

In this section we give a metrical Diophantine analysis for tame parabolic finite iterated function systems. In the first subsection we calculate the Hausdorff dimensions of certain subsets of the limit set that are of zero $h$ conformal measure. These sets are comprised of radial elements that under the system have a rather rapid approach to the parabolic points. In particular, these sets are the natural analogues of the sets of well-approximable numbers. In the second subsection we derive various limit laws that give useful approximations of the 'essential support' of the $h$-conformal measure associated with a tame finite parabolic iterated function system. Subsequently, we show that these laws lead to good estimates on the growth of the function $\zeta$ in the global formula (Theorem 3.5), which in turn give rise to a refined description of the conformal measure in terms of Hausdorff measures and packing measures with respect to some explicit gauge functions.

4.1. Iterated function systems in the spirit of Jarník. We first have to introduce the notion of a canonical ball. For $i \in \Omega, \delta>0$ and a hyperbolic word $\omega \in I^{*}$, we define

$$
\begin{aligned}
& B_{\omega}(i)=B_{\omega}=\bar{B}\left(\phi_{\omega}\left(x_{i}\right), R\left|\phi_{\omega}^{\prime}\left(x_{i}\right)\right|\right), \\
& B_{\omega}^{\delta}(i)=B_{\omega}^{\delta}=\bar{B}\left(\phi_{\omega}\left(x_{i}\right),\left(R\left|\phi_{\omega}^{\prime}\left(x_{i}\right)\right|\right)^{1+\delta}\right) .
\end{aligned}
$$

The closed ball $B_{\omega}$ will be referred to as the canonical ball associated with the hyperbolic word $\omega$.

Our main interest in this section will be focused on the sets

$$
J_{i}^{\delta}:=\bigcap_{q \geq 1} \bigcup_{n \geq q} \bigcup_{|\omega|=n} B_{\omega}^{\delta}(i)
$$

and

$$
J^{\delta}:=\bigcup_{i \in \Omega} J_{i}^{\delta}
$$

The main result in this section is stated in the following theorem. The proof of this theorem will occupy the remaining part of this section. It will be given in several steps, some of which are formulated in separate lemmata.

Theorem 4.1. Let $S=\left\{\phi_{i}: i \in I\right\}$ be a tame parabolic finite iterated function system satisfying (SSOSC). Then, for every $i \in \Omega$ the following hold:

(a) If $h \leq 1$, then

$$
\operatorname{HD}\left(J^{\delta}\right)=\frac{h}{1+\delta} .
$$


(b) If $h \geq 1$, then

$$
\operatorname{HD}\left(J_{i}^{\delta}\right)= \begin{cases}\frac{h}{1+\delta} & \text { if } \delta \geq h-1, \\ \frac{h+\delta p_{i}}{1+\delta\left(1+p_{i}\right)} & \text { if } \delta \leq h-1 .\end{cases}
$$

In particular, with $p_{\min }:=\min \left\{p_{i}: i \in \Omega\right\}$, we have

$$
\operatorname{HD}\left(J^{\delta}\right)= \begin{cases}\frac{h}{1+\delta} & \text { if } \delta \geq h-1, \\ \frac{h+\delta p_{\text {min }}}{1+\delta\left(1+p_{\min }\right)} & \text { if } \delta \leq h-1 .\end{cases}
$$

The first step in the proof is to give an upper bound for $\operatorname{HD}\left(J_{i}^{\delta}\right)$.

Lemma 4.2. For each $i \in \Omega$ and every $\delta>0$ we have

$$
\operatorname{HD}\left(J_{i}^{\delta}\right) \leq \min \left\{\frac{h}{1+\delta}, \frac{h+\delta p_{i}}{1+\delta\left(1+p_{i}\right)}\right\} .
$$

Proof. For $n \geq 1$, let $H_{n}$ denote the family of all hyperbolic words of length $n$. For every $\epsilon>0$ we have

$$
\begin{aligned}
\mathrm{H}^{\frac{h}{1+\delta}+\epsilon}\left(J_{i}^{\delta}\right) & \left.\leq \liminf _{q \rightarrow \infty} \sum_{n \geq q} \sum_{\omega \in H_{n}}\left(\left(R\left|\phi_{\omega}^{\prime}\left(x_{i}\right)\right|\right)^{1+\delta}\right)\right)^{\frac{h}{1+\delta}+\epsilon} \\
& \leq R^{h+\epsilon(1+\delta)} \liminf _{q \rightarrow \infty} \sum_{n \geq q} \sum_{\omega \in H_{n}}\left|\phi_{\omega}^{\prime}\left(x_{i}\right)\right|^{h+\epsilon(1+\delta)} .
\end{aligned}
$$

From Lemma 4.3 and Theorem 4.6 in [MU1] we deduce that there exists an $(h+\epsilon(1+\delta))$-semiconformal measure $\nu$. We then apply Theorem 5.1 in [MU1], which gives that $\nu$ is in fact $(h+\epsilon(1+\delta))$-conformal, and that $\nu\left(x_{j}\right)>0$ for some $j \in \Omega$. From the definition of the limit set $J$ it follows that there exists a hyperbolic word $\tau \in I^{*}$ such that $\phi_{\tau}\left(x_{j}\right) \in B\left(x_{i}, R\right)$. Hence, by Lemma 2.2, we have

$$
\left|\phi_{\omega}^{\prime}\left(x_{i}\right)\right| \leq K\left|\phi_{\omega}^{\prime}\left(\phi_{\tau}\left(x_{j}\right)\right)\right|=K\left|\phi_{\tau}^{\prime}\left(x_{j}\right)\right|^{-1}\left|\phi_{\omega \tau}^{\prime}\left(x_{j}\right)\right|
$$

for every hyperbolic word $\omega \in I^{*}$. Combining this estimate with the conformality of $\nu$, it follows that for each $q \geq 0$ and every $n \geq q$ we have

$$
\begin{aligned}
\sum_{n \geq q} \sum_{\omega \in H_{n}}\left|\phi_{\omega}^{\prime}\left(x_{i}\right)\right|^{h+\epsilon(1+\delta)} & \leq\left(K\left|\phi_{\tau}^{\prime}\left(x_{j}\right)\right|^{-1}\right)^{h+\epsilon(1+\delta)} \sum_{n \geq q} \sum_{\omega \in H_{n}}\left|\phi_{\omega \tau}^{\prime}\left(x_{j}\right)\right|^{h+\epsilon(1+\delta)} \\
& \leq \sum_{n \geq q} \sum_{|\omega|=n} \nu\left(\phi_{\omega \tau}\left(x_{j}\right)\right) \nu\left(x_{j}\right)^{-1} \\
& \leq \nu\left(x_{j}\right)^{-1} \nu\left(\left\{\phi_{\gamma}\left(x_{j}\right):|\gamma| \geq q+|\tau|\right\}\right) \leq \nu\left(x_{j}\right)^{-1} .
\end{aligned}
$$


Hence, $\mathrm{H}^{\frac{h}{1+\delta}}+\epsilon\left(J_{i}^{\delta}\right) \leq \nu\left(x_{j}\right)^{-1}$, and consequently $\operatorname{HD}\left(J_{i}^{\delta}\right) \leq \frac{h}{1+\delta}+\epsilon$. By letting $\epsilon$ tend to 0 , we derive that $\operatorname{HD}\left(J_{i}^{\delta}\right) \leq \frac{h}{1+\delta}$.

In order to obtain the second upper bound, note that by Lemma 2.4 the intersection $J \cap B\left(\phi_{\omega}\left(x_{i}\right),\left(R\left|\phi_{\omega}^{\prime}\left(x_{i}\right)\right|\right)^{1+\delta}\right)$ is contained in a central cone with vertex $\phi_{\omega}\left(x_{i}\right)$ and angle $\left.\leq C_{2} R^{1+\delta}\left|\phi_{\omega}^{\prime}\left(x_{i}\right)\right|\right)^{\delta p_{i}} \asymp\left(R\left|\phi_{\omega}^{\prime}\left(x_{i}\right)\right|\right)^{\delta p_{i}}$, or equivalently the radius of the base $\preceq\left(R\left|\phi_{\omega}^{\prime}\left(x_{i}\right)\right|\right)^{1+\delta\left(p_{i}+1\right)}$. Thus $J \cap$ $B\left(\phi_{\omega}\left(x_{i}\right),\left(R\left|\phi_{\omega}^{\prime}\left(x_{i}\right)\right|\right)^{1+\delta}\right)$ can be covered by at most const $\left(R\left|\phi_{\omega}^{\prime}\left(x_{i}\right)\right|\right)^{-\delta p_{i}}$ balls of radii $\left(R\left|\phi_{\omega}^{\prime}\left(x_{i}\right)\right|\right)^{1+\delta\left(p_{i}+1\right)}$. Therefore, for every $\epsilon>0$ we have

$$
\begin{aligned}
\mathrm{H}^{\frac{h+\delta p_{i}}{1+\delta\left(1+p_{i}\right)}+\epsilon} & \left(J_{i}^{\delta}\right) \\
& \leq \liminf _{q \rightarrow \infty} \sum_{n \geq q} \sum_{\omega \in H_{n}}\left(\left(R\left|\phi_{\omega}^{\prime}\left(x_{i}\right)\right|\right)^{1+\delta\left(1+p_{i}\right)}\right)^{\frac{h+\delta p_{i}}{1+\delta\left(1+p_{i}\right)}+\epsilon}\left(R\left|\phi_{\omega}^{\prime}\left(x_{i}\right)\right|\right)^{-\delta p_{i}} \\
& \asymp \liminf _{q \rightarrow \infty} \sum_{n \geq q} \sum_{\omega \in H_{n}}\left|\phi_{\omega}^{\prime}\left(x_{i}\right)\right|^{h+\epsilon\left(1+\delta\left(1+p_{i}\right)\right)} .
\end{aligned}
$$

Now the proof follows exactly in the same way as in the first part.

As a first step towards the proof of the lower bound in Theorem 4.1, we obtain the following lemma:

Lemma 4.3. There exists a universal constant $b(d) \geq 1$ such that the following holds: for every open set $G \subset \operatorname{Int} X$ and each $n \geq 1$, there exists a finite set $I_{G, n} \subset \bigcup_{j \geq n} I^{j}$ of mutually incomparable hyperbolic words, which has the properties that $m\left(\bigcup_{\omega \in I_{G, n}} B_{\omega}\right) \geq b(d)^{-1} m(G)$ and that the balls in $\left\{B_{\omega}: \omega \in I_{G, n}\right\}$ are pairwise disjoint subsets of $G$.

Proof. Fix $i \in \Omega$. We define

$J_{\infty}:=\pi\left(\left\{\omega \in I^{\infty} \backslash\left\{\tau i^{\infty}: \tau \in I^{*}\right\}: \omega\right.\right.$ contains arbitrarily long blocks of $i$ 's $\left.\}\right)$.

Then, since the conformal measure $m$ is positive on nonempty open subsets of $J$, Corollary 5.11 in [MU1] implies that $m\left(J_{\infty}\right)=1$. Now, let $q \geq 1$ be sufficiently large such that $\phi_{i^{q}}(X) \subset B\left(x_{i}, K^{-1} R\right)$. It follows from the definition of $J_{\infty}$ that if $x \in J_{\infty}$, then there exists an increasing infinite sequence $\left\{l_{j}\right\}_{j}$ with $l_{j} \geq n$ for all $j \geq 1$, a sequence $\left\{q_{j}\right\}_{j}$ with $q_{j} \geq q+1$ for all $j \geq 1$, and words $\omega^{(j)} \in I^{l_{j}+q_{j}}$ such that for all $j \geq 1$ we have $x \in \phi_{\omega^{(j)}}(X), \omega_{l_{j}}^{(j)} \neq i$ and $\left.\sigma^{l_{j}} \omega\right|_{q_{j}}=i^{q_{j}}$. It now follows that

$$
x \in \phi_{\left.\omega^{(j)}\right|_{l_{j}+1}}\left(B\left(x, K^{-1} R\right)\right) \subset B_{\omega^{(j)}},
$$

that $\left.\omega^{(j)}\right|_{l_{j}+1}$ is a hyperbolic word, and that $\lim _{j \rightarrow \infty} \operatorname{diam}\left(B_{\omega^{(j)}}\right)=0$. Hence, the set $G \cap J_{\infty}$ can be covered by canonical balls $B_{\omega}$ for which $|\omega| \geq n$. Let $\Gamma$ denote such a cover of $G \cap J_{\infty}$. By the Besicovitch Covering Theorem, there exists a universal constant $b(d) \geq 2$ such that $\Gamma$ contains $b(d) / 2$ subfamilies, each consisting of pairwise disjoint elements, such that $G$ is 
contained in the union of all balls in these subfamilies. It follows that for at least one of these subfamilies, say $\Gamma_{0}$, we have $m\left(\bigcup_{B_{\omega} \in \Gamma_{0}} B_{\omega}\right) \geq$ $2 / b(d) m\left(G \cap J_{\infty}\right)=2 / b(d) m(G)$. Since there clearly exists a finite subset $\Gamma_{f}$ of $\Gamma_{0}$ having the property that $m\left(\bigcup_{B_{\omega} \in \Gamma_{f}} B_{\omega}\right) \geq \frac{1}{2} m\left(\bigcup_{B_{\omega} \in \Gamma_{0}} B_{\omega}\right)$, the conclusion of the lemma follows.

Proof of Theorem 4.1. Our next step in the proof of the theorem is the construction of a Cantor set contained in $J_{i}^{\delta}$. Crucial for this will be a certain increasing sequence $\left\{n_{l}\right\}_{l \geq 0}$ of nonnegative integers, and it will become clear during the construction how one has to choose this sequence. We begin by defining for $l \geq 0$ the sets $I_{l} \subset I^{*}$ by induction as follows: let $B_{\emptyset}:=\bar{B}\left(x_{i}, R\right)$ and $I_{0}:=\{\emptyset\}$. Suppose that $I_{l}$ has been defined, and let $\omega \in I_{l}$ be fixed. By Lemma 4.3 there exists a finite set $\omega^{*}$ consisting of hyperbolic words such that

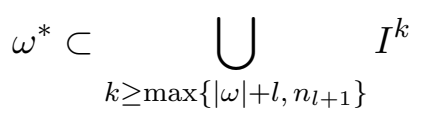

and the family $\left\{B_{\tau}\right\}_{\tau \in \omega^{*}}$ consists of pairwise disjoint balls such that $B_{\tau} \subset$ Int $B_{\omega}^{\delta}$ for every $\tau \in \omega^{*}$ (note that $\left.\tau\right|_{|\omega|}=\omega$ ). In addition

$$
m\left(\bigcup_{\tau \in \omega^{*}} B_{\tau}\right) \geq \frac{1}{b(d)} m\left(\operatorname{Int} B_{\omega}^{\delta}\right) \gg m\left(B_{\omega}^{\delta}\right) .
$$

Here, the latter inequality follows from the conformality of $m$, Lemma 2.2 and Corollary 3.3. Put

$$
I_{l+1}=\bigcup_{\omega \in I_{l}} \omega^{*}
$$

Now, let $\left\{F_{l}\right\}_{l \geq 1}$ denote the family of nested nonempty compact subsets of $B_{\emptyset}$ given by

$$
F_{l}:=\bigcap_{\omega \in I_{l}} B_{\omega} .
$$

Note that we have in particular that

$$
F=\bigcap_{l \geq 1} F_{l} \neq \emptyset
$$

Next, for each $l \geq 1$ we construct a Borel probability measure $\mu_{l}$ supported on the set $F_{l-1}$ as follows: let $\mu_{1}:=\left.\frac{1}{m\left(B_{\emptyset}\right)} m\right|_{B_{\emptyset}}$, and assume that the measure $\mu_{l}$ has already been defined for some $l \geq 1$. Recall that

$$
\omega^{*}:=\left\{\tau \in I_{l+1}:\left.\tau\right|_{n_{l}}=\omega\right\}
$$

for $\omega \in I_{l}$. Now, for each $\omega \in I_{l}$ and every Borel set $A \subset B_{\omega}$ we put

$$
\mu_{l+1}(A):=\frac{\sum_{\tau \in \omega^{*}} m\left(A \cap B_{\tau}\right)}{\sum_{\tau \in \omega^{*}} m\left(B_{\tau}\right)} .
$$


This defines a Borel probability measure $\mu_{l+1}$ on $F_{l}$ having the property that $\mu_{l+1}\left(B_{\omega}\right)=\mu_{l}\left(B_{\omega}\right)$ for every $\omega \in I_{l}$. A straightforward inductive argument gives that $\mu_{q}\left(B_{\omega}\right)=\mu_{l}\left(B_{\omega}\right)$ for every $q \geq l$. Also, since for each $\omega \in \bigcup_{l>0} I_{l}$ the set $B_{\omega} \cap F$ is an open subset of $F$, we conclude that the weak limit $\mu:=\lim _{l \rightarrow \infty} \mu_{l}$ exists and is supported on $F$, and that $\mu\left(B_{\omega}\right)=\mu_{l}\left(B_{\omega}\right)$ for each $l \geq 1$ and every $\omega \in I_{l}$. For $\omega \in I_{l}$ and $j \leq l$, let $k_{j}=k_{j}(\omega) \leq|\omega|$ denote the unique integer determined by $\left.\omega\right|_{k_{j}} \in I_{j}$. Using (4.1) and (4.2), a straightforward inductive argument gives that for every $l \geq 1$ and every $\omega \in I_{l}$ we have

$$
\begin{aligned}
\mu\left(B_{\omega}\right) & =\mu_{l}\left(B_{\omega}\right)=\prod_{j=1}^{l} \frac{m\left(B_{\left.\omega\right|_{k_{j}}}\right)}{\sum_{\left.\tau \in \omega\right|_{k_{j}-1} ^{*}} m\left(B_{\tau}\right)} \\
& =m\left(B_{\omega}\right) \prod_{j=1}^{l-1} \frac{m\left(B_{\left.\omega\right|_{k_{j}}}\right)}{\sum_{\left.\tau \in \omega\right|_{k_{j}} ^{*}} m\left(B_{\tau}\right)} \frac{1}{m\left(B\left(x_{i}, R\right)\right)} \\
& =m\left(B_{\omega}\right) \prod_{j=1}^{l-1} \frac{m\left(B_{\left.\omega\right|_{k_{j}}}\right)}{m\left(B_{\left.\omega\right|_{k_{j}}}^{\delta}\right)} \exp (O(l)) \\
& =m\left(B_{\omega}\right) \prod_{j=1}^{l-1} \frac{\left|\phi_{\left.\omega\right|_{k_{j}}}^{\prime}\left(x_{i}\right)\right|^{h}}{\left|\phi_{\left.\omega\right|_{k_{j}}}^{\prime}\left(x_{i}\right)\right|^{h} m\left(B\left(x_{i},\left(R\left|\phi_{\left.\omega\right|_{k_{j}}}^{\prime}\left(x_{i}\right)\right|\right)^{\delta}\right)\right)} \exp (O(l)) \\
& =m\left(B_{\omega}\right) \prod_{j=1}^{l-1} m\left(B\left(x_{i},\left(R\left|\phi_{\left.\omega\right|_{k_{j}}}^{\prime}\left(x_{i}\right)\right|\right)^{\delta}\right)\right)^{-1} \exp (O(l)) .
\end{aligned}
$$

For every $\eta \in \bigcup_{j \geq l-1} I_{j}$ define

$$
\prod_{l-1}(\eta):=\prod_{j=1}^{l-1} m\left(B\left(x_{i},\left(R\left|\phi_{\left.\eta\right|_{k_{j}}}^{\prime}\left(x_{i}\right)\right|\right)^{\delta}\right)\right)^{-1} .
$$

Since

$$
\lim _{n \rightarrow \infty} \sup \left\{\left|\phi_{\omega}^{\prime}\left(x_{i}\right)\right|: \omega \in I^{n}\right\}=0,
$$

it follows that there exists $n_{0} \geq 1$ such that for each $\omega$ with $|\omega| \geq n_{0}$ we have

$$
\left(R\left|\phi_{\omega}^{\prime}\left(x_{i}\right)\right|\right)^{1+\delta} \leq \frac{1}{3} R\left|\phi_{\omega}^{\prime}\left(x_{i}\right)\right| .
$$

Since the set $I_{l}$ is finite, it follows from (4.4) that there exists a positive number $\widetilde{R} \leq R$ such that if $\omega \in I_{l}$ and if $R\left|\phi_{\tau}^{\prime}\left(x_{i}\right)\right| \leq \widetilde{R}$ for some $\tau \in \omega^{*}$, then $|\omega| \geq n_{0}$. For fixed $z \in F$ and $0<r \leq \widetilde{R} / 3$, consider the family $\mathcal{F}$ of all words $\omega \in \bigcup_{l \geq 0} I_{l+1}$ for which

$$
B_{\omega} \cap B(z, r) \cap J \neq \emptyset, \quad R\left|\phi_{\omega}^{\prime}\left(x_{i}\right)\right|<3 r, \quad R\left|\phi_{\left.\omega\right|_{k_{l}}}^{\prime}\left(x_{i}\right)\right| \geq 3 r .
$$


We shall now see that the family $\mathcal{F}_{*}=\left\{\left.\omega\right|_{k_{l}}: \omega \in \mathcal{F}\right\}$ is a singleton, and that if this is the case with $\{\gamma\}=\mathcal{F}_{*}$, then it follows that

$$
B(z, r) \subset B_{\gamma} .
$$

For this, fix some element $\gamma \in \mathcal{F}_{*}$ and $\omega \in \mathcal{F}$ such that $\gamma=\left.\omega\right|_{k_{l}}$ and such that $y \in B_{\omega} \cap B(z, r) \cap J$. Clearly, by construction of the set $J$, we have $y \in B_{\gamma}^{\delta}$. From (4.5) and (4.6) we deduce that if $x \in B(z, r)$, then

$$
\begin{aligned}
\left\|x-\phi_{\gamma}\left(x_{i}\right)\right\| & \leq\|x-z\|+\|z-y\|+\left\|y-\phi_{\gamma}\left(x_{i}\right)\right\| \\
& <r+r+\left(R\left|\phi_{\omega}^{\prime}\left(x_{i}\right)\right|\right)^{1+\delta} \\
& \leq 2 r+\frac{1}{3} R\left|\phi_{\omega}^{\prime}\left(x_{i}\right)\right| \leq \frac{2}{3} R\left|\phi_{\left.\omega\right|_{k_{l}}}^{\prime}\left(x_{i}\right)\right|+\frac{1}{3} R\left|\phi_{\left.\omega\right|_{k_{l}}}^{\prime}\left(x_{i}\right)\right| \\
& =R\left|\phi_{\omega}^{\prime}\left(x_{i}\right)\right|=R\left|\phi_{\gamma}^{\prime}\left(x_{i}\right)\right| .
\end{aligned}
$$

Hence we have proved (4.7); in particular, using (4.6) and the construction of the set $F$, we obtain $\mathcal{F}_{*}=\{\gamma\}$.

Let $\epsilon>0$ be fixed. Since $T_{0}:=\sup \left\{\prod(\tau): \tau \in I_{l-1}\right\}<\infty$, we obtain for $n_{l}$ sufficiently large and for all $\eta \in I_{l}$ that

$$
T_{0} \exp (O(l)) \leq\left|\phi_{\eta}^{\prime}\left(x_{i}\right)\right|^{-\epsilon} .
$$

Combining this estimate and (4.6), it follows that

$$
\prod_{l-1}(\gamma) \exp (O(l)) \leq r^{-\epsilon}
$$

To complete the proof of Theorem 4.1, it now suffices to show that $\mu(B(z, r))$ can essentially be estimated from above by $r^{-2 \epsilon} r^{\theta}$, for

$$
\theta=\frac{h}{1+\delta} \quad \text { or } \quad \theta=\frac{h+\delta p_{i}}{1+\delta\left(1+p_{i}\right)}
$$

We split this estimate into three cases:

Case 1: $\quad r \geq\left(R\left|\phi_{\gamma}^{\prime}\left(x_{i}\right)\right|\right)^{1+\delta}$. Using (4.7), (4.8) and the conformality of $m$, we obtain

$$
\begin{aligned}
& \mu(B(z, r)) \leq \mu\left(B_{\gamma}\right)=\mu_{l}\left(B_{\gamma}\right) \\
& \leq m\left(B_{\gamma}\right) \prod_{l-1}(\gamma) \exp (O(l))=\left|\phi_{\gamma}^{\prime}\left(x_{i}\right)\right|^{h} \prod_{l-1}(\gamma) \exp (O(l)) \\
& \ll\left|\phi_{\gamma}^{\prime}\left(x_{i}\right)\right|^{h} r^{-\epsilon} \ll r^{\frac{h}{1+\delta}-\epsilon},
\end{aligned}
$$

which completes the discussion for this case. 
Before dealing with the remaining cases, note that, using (4.3), (4.6), (4.8) and Corollary 3.3, we have

$$
\begin{aligned}
\mu(B(z, r)) & \leq \sum_{\omega \in \mathcal{F}} \mu\left(B_{\omega}\right)=\sum_{\omega \in \mathcal{F}} m\left(B_{\omega}\right) \prod_{l}(\omega) \exp (O(l)) \\
& \leq m(B(z, 7 r)) \prod_{l}(\omega) \exp (O(l)) \\
& =m(B(z, 7 r)) \prod_{l-1}(\omega) \exp \left(O(l)\left(m\left(B\left(x_{i},\left(R\left|\phi_{\gamma}^{\prime}\left(x_{i}\right)\right|\right)^{\delta}\right)\right)^{-1}\right)\right. \\
& \ll r^{-\epsilon}\left|\phi_{\gamma}^{\prime}\left(x_{i}\right)\right|^{-\delta\left(h+(h-1) p_{i}\right)} m(B(z, 7 r)) .
\end{aligned}
$$

Case 2: $\quad r \leq\left(R\left|\phi_{\gamma}^{\prime}\left(x_{i}\right)\right|\right)^{1+\delta}$ and $r \geq K^{2} R Q^{p_{i}+2}\left(R\left|\phi_{\gamma}^{\prime}\left(x_{i}\right)\right|\right)^{1+\delta+\delta p_{i}}$. From (4.7) and Lemma 2.2 we deduce that

$$
r \leq R\left|\phi_{\gamma}^{\prime}\left(x_{i}\right)\right| \leq K R\left|\phi_{\left.\tau\right|_{n}}^{\prime}\left(\pi\left(\sigma^{n} \tau\right)\right)\right|,
$$

where $z=\pi(\tau)$ and $\left.\tau\right|_{n}=\gamma$. This implies that

$$
r / K \leq R\left|\phi_{\left.\tau\right|_{n}}^{\prime}\left(\pi\left(\sigma^{n} \tau\right)\right)\right|=R\left|\phi_{\gamma}^{\prime}\left(\pi\left(\sigma^{n} \tau\right)\right)\right| .
$$

Now, since $z \in B_{\gamma} \cap J$, we have $z \in B_{\gamma}^{\delta}$, and therefore

$$
\pi\left(\sigma^{n} \tau\right) \in B\left(x_{i}, K\left(R\left|\phi_{\gamma}^{\prime}\left(x_{i}\right)\right|\right)^{\delta}\right) .
$$

Let $\sigma^{n} \tau=i^{q} \omega$, with $\omega_{1} \neq i$. By Proposition 3.1 (formula (3.2)), we have $\left\|\pi\left(\sigma^{n} \tau\right)-x_{i}\right\| \geq Q^{-1} q^{-\frac{1}{p_{i}}}$. Hence, using the fact that $Q^{-1} q^{-\frac{1}{p_{i}}} \leq$ $K\left(R\left|\phi_{\gamma}^{\prime}\left(x_{i}\right)\right|\right)^{\delta}$ and Proposition 3.1 (formula (3.1)), we obtain

$$
\begin{aligned}
R\left|\phi_{\tau \mid n+q+1}^{\prime}\left(\pi\left(\sigma^{n+q+1} \tau\right)\right)\right| & =R\left|\phi_{\gamma}^{\prime}\left(\pi\left(\sigma^{n} \tau\right)\right)\right| \cdot\left|\phi_{i^{q} \omega_{1}}^{\prime}(\pi(\sigma \omega))\right| \\
& \leq R\left|\phi_{\gamma}^{\prime}\left(\pi\left(\sigma^{n} \tau\right)\right)\right| Q q^{-\frac{1}{p_{i}}} \\
& \leq K R Q^{p_{i}+2}\left(R\left|\phi_{\gamma}^{\prime}\left(x_{i}\right)\right|\right)^{1+\delta\left(p_{i}+1\right)} \\
& \leq r / K .
\end{aligned}
$$

It follows that

$$
(r / K)^{*}=R\left|\phi_{\gamma}^{\prime}\left(\pi\left(\sigma^{n} \tau\right)\right)\right|, \quad(r / K)_{*}=R\left|\phi_{\left.\tau\right|_{n+q+1}}^{\prime}\left(\pi\left(\sigma^{n+q+1} \tau\right)\right)\right| .
$$

Choose a small $\kappa>0$ to be specified in the course of the proof. Without loss of generality we may assume that $z \notin J_{i}^{\delta+\kappa}$. Thus by choosing $r>0$ to be sufficiently small, we can assume that $z \notin B_{\gamma}^{\delta+\kappa}$, and hence in particular that $\pi\left(\sigma^{n} \tau\right) \notin B\left(x_{i}, K^{-1}\left(R\left|\phi_{\gamma}^{\prime}\left(x_{i}\right)\right|\right)^{\delta+\kappa}\right)$. Since

$$
\left\|\pi\left(\sigma^{n} \tau\right)-x_{i}\right\| \leq Q q^{-\frac{1}{p_{i}}}
$$


by Proposition 3.1 (formula (3.2)), we have $Q q^{-\frac{1}{p_{i}}} \geq K^{-1}\left(R\left|\phi_{\gamma}^{\prime}\left(x_{i}\right)\right|\right)^{\delta+\kappa}$. Hence, using Proposition 3.1 (formula (3.1)), we obtain

$$
\begin{aligned}
R\left|\phi_{\left.\tau\right|_{n+q+1}}^{\prime}\left(\pi\left(\sigma^{n+q+1} \tau\right)\right)\right| & =R\left|\phi_{\gamma}^{\prime}\left(\pi\left(\sigma^{n} \tau\right)\right)\right| \cdot\left|\phi_{i^{q} \omega_{1}}^{\prime}(\pi(\sigma \omega))\right| \\
& \geq K^{-1} R\left|\phi_{\gamma}^{\prime}\left(x_{i}\right)\right| Q^{-1} q^{-\frac{1}{p_{i}}} \\
& \geq\left(R(K Q)^{p_{i}+2}\right)^{-1}\left(R\left|\phi_{\gamma}^{\prime}\left(x_{i}\right)\right|\right)^{1+(\delta+\kappa)\left(p_{i}+1\right)} .
\end{aligned}
$$

Write $r=c\left(R\left|\phi_{\gamma}^{\prime}\left(x_{i}\right)\right|\right)^{1+\delta+\eta}$, for $0 \leq \eta \leq \delta p_{i}$ and $1 \leq c \leq K^{2} R Q^{p_{i}+2}$. Suppose first that the first part of the global formula (Theorem 3.5) holds for the centre $z$ and radius $r / K$. Using (4.12), we obtain

$$
\begin{aligned}
& c K^{-1}\left(R\left|\phi_{\gamma}^{\prime}\left(x_{i}\right)\right|\right)^{1+\delta+\eta} \\
& \geq R\left|\phi_{\gamma}^{\prime}\left(\pi\left(\sigma^{n} \tau\right)\right)\right|\left(\frac{R\left|\phi_{\tau \mid n+q+1}^{\prime}\left(\pi\left(\sigma^{n+q+1} \tau\right)\right)\right|}{R\left|\phi_{\gamma}^{\prime}\left(\pi\left(\sigma^{n} \tau\right)\right)\right|}\right)^{\frac{1}{p_{i}+1}} \\
& \geq R K^{-1}\left|\phi_{\gamma}^{\prime}\left(x_{i}\right)\right|\left(\left(R(K Q)^{p_{i}+2}\right)^{-1} \frac{\left(R\left|\phi_{\gamma}^{\prime}\left(x_{i}\right)\right|\right)^{1+(\delta+\kappa)\left(p_{i}+1\right)}}{R\left|\phi_{\gamma}^{\prime}\left(\pi\left(\sigma^{n} \tau\right)\right)\right|}\right)^{\frac{1}{p_{i}+1}} \\
& \asymp\left|\phi_{\gamma}^{\prime}\left(x_{i}\right)\right|^{1+\delta+\kappa} .
\end{aligned}
$$

Note that if $r>0$ is chosen to be sufficiently small (and hence the word length of $\gamma$ is large), we have $\eta \leq 2 \kappa$. Then, applying Theorem 3.5, (4.9) and Corollary 3.7, we obtain

$$
\begin{aligned}
\mu(B(z, r)) & \ll r^{-\epsilon}\left|\phi_{\gamma}^{\prime}\left(x_{i}\right)\right|^{-\delta\left(h+(h-1) p_{i}\right)} m(B(z, r / K)) \\
& \asymp r^{-\epsilon} r^{h}\left|\phi_{\gamma}^{\prime}\left(x_{i}\right)\right|^{-\delta\left(h+(h-1) p_{i}\right)}\left(\frac{r / K}{(r / K)^{*}}\right)^{h-1} \\
& \asymp r^{-\epsilon} r^{h}\left|\phi_{\gamma}^{\prime}\left(x_{i}\right)\right|^{-\delta\left(h+(h-1) p_{i}\right)}\left(\frac{r}{\left|\phi_{\gamma}^{\prime}\left(x_{i}\right)\right|}\right)^{(h-1) p_{i}} \\
& =r^{-\epsilon} r^{h+(h-1) p_{i}}\left|\phi_{\gamma}^{\prime}\left(x_{i}\right)\right|^{-\delta\left(h+(h-1) p_{i}\right)-(h-1) p_{i}} \\
& \asymp r^{-\epsilon} r^{h+(h-1) p_{i}} r^{\frac{-\delta\left(h+(h-1) p_{i}\right)-(h-1) p_{i}}{1+\delta+\eta}}=r^{-\epsilon} r^{\frac{h p_{i} \eta-p \eta+h+h \eta}{1+\delta+\eta}} .
\end{aligned}
$$

Note that we have

$$
\frac{h p_{i} \eta-p \eta+h+h \eta}{1+\delta+\eta} \geq \frac{h}{1+\delta}-\epsilon
$$

if and only if

$$
\eta\left(h p_{i}-p_{i}+h p_{i} \delta-p_{i} \delta+h \delta\right) \geq-\epsilon(1+\delta+\eta) .
$$

Clearly, since $\eta \leq 2 \kappa$, the latter inequality is satisfied if we choose $\kappa>0$ to be sufficiently small. Hence, we can assume without loss of generality that 
(4.13) holds. It then follows that

$$
\mu(B(z, r)) \leq r^{\frac{h}{1+\delta}-2 \epsilon},
$$

which gives the Case 2 assuming the first part of the global formula.

Now suppose that the second part of the global formula (Theorem 3.5) holds for the centre $z=\pi(\tau)$ and radius $r / K$. Then (4.9), Corollary 3.7 and Theorem 3.5 imply that

$$
\begin{aligned}
\mu(B(z, r)) & \ll r^{-\epsilon}\left|\phi_{\gamma}^{\prime}\left(x_{i}\right)\right|^{-\delta\left(h+(h-1) p_{i}\right)} m(B(z, r / K)) \\
& \leq r^{-\epsilon}\left|\phi_{\gamma}^{\prime}\left(x_{i}\right)\right|^{-\delta\left(h+(h-1) p_{i}\right)} r^{h}\left(\frac{(r / K)_{*}}{r}\right)^{h-1} \\
& \asymp r^{-\epsilon} r\left|\phi_{\gamma}^{\prime}\left(x_{i}\right)\right|^{-\delta\left(h+(h-1) p_{i}\right) p}\left|\phi_{\left.\tau\right|_{n+q+1} ^{\prime}}^{\prime}\left(\pi\left(\sigma^{n+q+1} \tau\right)\right)\right|^{h-1} .
\end{aligned}
$$

If $h \leq 1$, then using (4.12), we can continue the estimate in this case as follows:

$$
\begin{aligned}
\mu(B(z, r)) & \ll r^{-\epsilon} r\left|\phi_{\gamma}^{\prime}\left(x_{i}\right)\right|^{-\delta\left(h+(h-1) p_{i}\right) p_{i}}\left|\phi_{\gamma}^{\prime}\left(x_{i}\right)\right|^{(h-1)(1+(\delta+\kappa)(p+1))} \\
& =r^{-\epsilon} r\left|\phi_{\gamma}^{\prime}\left(x_{i}\right)\right|^{h+h \kappa p_{i}+h \kappa-\kappa p_{i}-1-\delta-\kappa} \\
& =r^{-\epsilon} r\left|\phi_{\gamma}^{\prime}\left(x_{i}\right)\right|^{h-1-\delta+a \kappa},
\end{aligned}
$$

where we have set $a:=h p_{i}+h-p_{i}-1 \leq 0$. Hence,

$$
\mu(B(z, r)) \ll r^{-\epsilon} r r^{\frac{h-1-\delta+a \kappa}{1+\delta+\eta}}=r^{-\epsilon} r^{\frac{h+\eta+a \kappa}{1+\delta+\eta}} \leq r^{-\epsilon} r^{\frac{h+a \kappa}{1+\delta}},
$$

where in the last inequality we used the assumption that $h \leq 1$. Now, by choosing $\kappa>0$ to be sufficiently small, it follows that

$$
\mu(B(z, r)) \leq r^{\frac{h}{1+\delta}-2 \epsilon} .
$$

This completes Case 2 for $h \leq 1$.

If $h>1$, then using (4.11), we can continue the estimate in (4.14) as follows:

$$
\begin{aligned}
\mu(B(z, r)) & \ll r^{-\epsilon} r\left|\phi_{\gamma}^{\prime}\left(x_{i}\right)\right|^{-\delta\left(h+(h-1) p_{i}\right)}\left|\phi_{\gamma}^{\prime}\left(x_{i}\right)\right|^{(h-1)(1+\delta(p+1))} \\
& =r^{-\epsilon} r\left|\phi_{\gamma}^{\prime}\left(x_{i}\right)\right|^{h-1-\delta}=r^{-\epsilon} r r^{\frac{h-1-\delta}{1+\delta+\eta}}=r^{-\epsilon} r^{\frac{h+\eta}{1+\delta+\eta}} \\
& \leq \begin{cases}r^{\frac{h}{1+\delta}}-\epsilon & \text { if } \delta \geq h-1 \\
r^{\frac{h+\delta p_{i}}{1+\delta\left(1+p_{i}\right)}} & \text { if } \delta \leq h-1 .\end{cases}
\end{aligned}
$$

Here, the latter inequality is obtained by using the facts that $\eta \leq \delta p_{i}$ and that for $\delta \leq h-1$ it holds that $\frac{h+\eta}{1+\delta+\eta}$ decreases if $\eta$ increases.

Hence, the proof of Case 2 is complete. 
Case 3: $\quad r \leq K^{2} R Q^{p_{i}+2}\left(R\left|\phi_{\gamma}^{\prime}\left(x_{i}\right)\right|\right)^{1+\delta+\delta p_{i}}$. From (4.9) and Corollary 3.7 we deduce that

$$
\begin{aligned}
\mu(B(z, r)) & \ll r^{-\epsilon}\left|\phi_{\gamma}^{\prime}\left(x_{i}\right)\right|^{-\delta\left(h+(h-1) p_{i}\right)} m(B(z, r / K)) \\
& =r^{-\epsilon}\left|\phi_{\gamma}^{\prime}\left(x_{i}\right)\right|^{-\delta\left(h+(h-1) p_{i}\right)} r^{h} \zeta(z, r / K) \\
& \ll r^{-\epsilon} r^{h} r^{-\frac{\delta\left(h+(h-1) p_{i}\right)}{1+\delta+\delta p_{i}}} \zeta(z, r / K) \\
& =r^{\frac{h+\delta p_{i}}{1+\delta+\delta p_{i}}-\epsilon} \zeta(z, r / K) .
\end{aligned}
$$

If $h \geq 1$, then we can apply Theorem 3.5, and we obtain

$$
\mu(B(z, r)) \ll r^{\frac{h+\delta p_{i}}{1+\delta+\delta p_{i}}-\epsilon} .
$$

If $h \leq 1$, we can assume $i=i_{\max }$, which means $p_{i}=\max \left\{p_{j}: j \in \Omega\right\}$. Let $k \geq 1$ be the index in the hyperbolic zoom associated with the point $z$ and with the radius $r / K$. If $n_{k+1}=n_{k}+1$, then we can proceed as in the previous case to obtain the desired result. Hence, suppose that $n_{k+1} \neq n_{k}+1$. It follows that $\sigma^{n_{k}} \tau=j^{u} \tau_{n_{k}+1}$ for some $j \in \Omega, u \geq 1$ and $\tau_{n_{k}+1} \neq j$. Now, for $t \in\left[(r / K)_{*},(r / K)^{*}\right]$ we write $\zeta(z, t)=t^{\alpha(t)}$. Then

$$
\begin{aligned}
\alpha(t) & =\frac{\log \zeta(z, t)}{\log t} \\
& = \begin{cases}p_{j}(h-1)+\frac{p_{j}(1-h) \log \left((r / K)^{*}\right)}{\log t} & \text { for }(r / K)^{*} \geq r \geq(r / K)^{*}\left(\frac{(r / K)_{*}}{(r / K)^{*}}\right)^{\frac{1}{p_{j}+1}}, \\
1-h+\frac{(h-1) \log \left((r / K)_{*}\right)}{\log t} & \text { for }(r / K)_{*} \leq r \leq(r / K)^{*}\left(\frac{(r / K)_{*}}{(r / K)^{*}}\right)^{\frac{1}{p_{j}+1}} .\end{cases}
\end{aligned}
$$

From this we deduce that $\alpha$ has its minimum at $t=(r / K)^{*}\left(\frac{(r / K)_{*}}{(r / K)^{*}}\right)^{\frac{1}{p_{j}+1}}$. Therefore, we can assume without loss of generality that

$$
(r / K)=(r / K)^{*}\left(\frac{(r / K)_{*}}{(r / K)^{*}}\right)^{\frac{1}{p_{j}+1}} .
$$

Also, by choosing $\kappa>0$ sufficiently small, we can assume that $z \notin J^{\delta+\kappa}$. For $r>0$ small, we then have $z \notin B_{\left.\tau\right|_{k}}^{\delta+\kappa}\left(p_{j}\right)$. Now, by the same arguments as those leading to formula (4.12) in Case 2, we have

$$
(r / K)_{*} \geq\left(R(K Q)^{p_{i}+2}\right)^{-1}\left((r / K)^{*}\right)^{1+(\delta+\kappa)\left(p_{j}+1\right)} .
$$

Hence, Theorem 3.5 and (4.16) imply that

$$
\zeta(z, r / K) \leq\left(\frac{(r / K)_{*}}{(r / K)^{*}}\right)^{\frac{(h-1) p_{j}}{p_{j}+1}} .
$$


Now write

$$
\left(\frac{(r / K)_{*}}{(r / K)^{*}}\right)^{\frac{(h-1) p_{j}}{p_{j}+1}}=(r / K)^{\alpha}=(r / K)^{*}\left(\left(\frac{(r / K)_{*}}{(r / K)^{*}}\right)^{\frac{1}{p_{j}+1}}\right)^{\alpha}
$$

and for every $t \in(0,1)$ consider the number $\alpha(t)$ determined by the equation

$$
\left(\frac{t}{(r / K)^{*}}\right)^{\frac{(h-1) p_{j}}{p_{j}+1}}=(r / K)^{*}\left(\left(\frac{t}{(r / K)^{*}}\right)^{\frac{1}{p_{j}+1}}\right)^{\alpha(t)} .
$$

We are interested in a sufficiently good lower bound on $\alpha(r / K)$. And indeed, solving Equation (4.19) for $\alpha(t)$, one easily deduces that the function $t \mapsto$ $\alpha(t)$ is increasing throughout the entire interval $(0,1)$. Therefore, invoking (4.17), we may assume that

$$
\begin{aligned}
(r / K)_{*} & \left.=R(K Q)^{p_{i}+2}\right)^{-1}\left((r / K)^{*}\right)^{1+(\delta+\kappa)\left(p_{j}+1\right)} \\
& \left.\leq R(K Q)^{p_{i}+2}\right)^{-1}\left((r / K)^{*}\right)^{1+\delta\left(p_{j}+1\right)} .
\end{aligned}
$$

Combining this and (4.16), we obtain

$$
(r / K) \ll(r / K)^{*}\left((r / K)^{*}\right)^{\delta}=\left((r / K)^{*}\right)^{1+\delta} .
$$

Then by combining this, (4.18) and (4.17), we get

$$
\zeta(z, r / K) \ll\left((r / K)^{*}\right)^{(\delta+\kappa) p_{j}(h-1)} \ll(r / K)^{\frac{p_{j}(h-1)(\delta+\kappa)}{1+\delta}} .
$$

Substituting this latter inequality in (4.15), we obtain

$$
\mu(B(z, r)) \ll r^{\frac{h+\delta p_{i}}{1+\delta+\delta p_{i}}+\frac{\delta p_{j}(h-1)}{1+\delta}} r^{-\epsilon+\frac{\kappa p_{j}(1-h)}{1+\delta}} .
$$
A straightforward calculation, using the facts that $p_{i} \geq p_{j}$ and $h \geq \frac{p_{j}}{p_{j}+1}$,
shows that

$$
\frac{h+\delta p_{i}}{1+\delta+\delta p_{i}}+\frac{\delta p_{j}(h-1)}{1+\delta} \geq \frac{h}{1+\delta} .
$$

Hence, if $\kappa$ is chosen sufficiently small, we finally obtain

$$
\mu(B(z, r)) \ll r^{\frac{h}{1+\delta}}-\epsilon .
$$

4.2. Limit laws for iterated function systems. Define the set

$$
I_{*}:=\left\{i^{n} j: i \in \Omega, j \neq i, n \geq 1\right\} \cup(I \backslash \Omega) .
$$

A word $\omega \in I^{\infty}$ can be written uniquely as an infinite word in elements from $I_{*}$ if and only if $\omega$ is not of the form $\tau i^{\infty}$ for any $i \in \Omega$ and $\tau \in I^{*}$. Let

$$
\sigma^{*}: I_{*}^{\infty} \rightarrow I_{*}^{\infty}
$$

denote the shift map on $I_{*}^{\infty}$. Also, for $i \in \Omega$ and $\omega \in I_{*}^{\infty}$ define

$$
Q_{i}(\omega):= \begin{cases}n & \text { if } \omega_{1}=i^{n} j \text { for some } n \geq 1 \text { and } j \neq i \\ 0 & \text { otherwise. }\end{cases}
$$


In [MU1] we proved that the iterated function system $S^{*}=\left\{\phi_{\omega}: \omega \in I^{*}\right\}$ is hyperbolic, and that $S^{*}$ is regular if and only if $S$ is regular. The shift map $\sigma^{*}$ can be interpreted as the symbolic representation of the system $S^{*}$. As in the previous section, in this section we shall always assume that $S$ is a tame parabolic finite iterated function system satisfying (SSOSC), and that $m$ is the associated conformal measure for $S$. Clearly, $m$ is also conformal for $S^{*}$. Hence, there exist Borel probability measures $\widetilde{m}$ and $\mu^{*}$ on $I_{*}^{\infty}$ that are equivalent to each other (with uniformly bounded Radon-Nikodým derivatives) such that $m=\widetilde{m} \circ \pi^{-1}$ and $\mu^{*} \circ\left(\sigma^{*}\right)^{-1}=\mu^{*}$ (see [MU1]). For $\epsilon \in \mathbb{R}, i \in \Omega$ and $n \geq 1$, we define

$$
A_{i, n}(\epsilon):=\left\{\omega \in I_{*}^{\infty}: Q_{i}(\omega) \geq n^{\frac{p_{i}}{h+(h-1) p_{i}}-\epsilon}\right\}
$$

and

$$
A_{i, \infty}(\epsilon):=\left\{\omega \in I_{*}^{\infty}: \sigma^{* n}(\omega) \in A_{i, n}(\epsilon) \text { for infinitely many } n\right\} .
$$

Lemma 4.4. For $i \in \Omega$ and $\epsilon \in \mathbb{R}$ we have $\widetilde{m}\left(A_{i, \infty}(\epsilon)\right)>0$ if and only if $\epsilon \geq 0$.

Proof. Using the definition of $\widetilde{m}$ and the conformality of $m$, we obtain

$$
\begin{aligned}
\sum_{n \geq 1} \mu^{*}\left(\left(\sigma^{*}\right)^{-n}\left(A_{i, n}(\epsilon)\right)\right) & =\sum_{n \geq 1} \mu^{*}\left(A_{i, n}(\epsilon)\right)=\sum_{n \geq 1} \widetilde{m}\left(A_{i, n}(\epsilon)\right) \\
& =\sum_{n \geq 1} \sum_{\substack{p_{i} \\
k \geq n^{h+(h-1) p_{i}}-\epsilon}} k^{-\frac{p_{i}+1}{p_{i}} h} \\
& \asymp \sum_{n \geq 1} n^{-1+\epsilon \frac{h+(h-1) p_{i}}{p_{i}}} .
\end{aligned}
$$

Since $h+(h-1) p_{i}>0$ (see [MU2]), it follows that the series

$$
\sum_{n \geq 1} \mu^{*}\left(\left(\sigma^{*}\right)^{-n}\left(A_{i, n}(\epsilon)\right)\right)
$$

converges for $\epsilon<0$. Thus, the "weaker part" of the Borel-Canteli lemma gives that $\mu_{*}\left(A_{i, \infty}(\epsilon)\right)=0$, which then implies that $\widetilde{m}\left(A_{i, \infty}(\epsilon)\right)=0$. This proves one direction of the equivalence in the lemma.

In order to prove the remaining part of the lemma, recall the following well-known result from elementary analysis:

- Let $\left(X_{n}\right)_{n \in \mathbb{N}}$ be a sequence of events in a probability space $(X, P)$. If $\sum_{n \in \mathbb{N}} P\left(X_{n}\right)=\infty$ and if $P\left(X_{n} \cap X_{k}\right) \ll P\left(X_{n}\right) P\left(X_{k}\right)$ for all distinct $n, k \in \mathbb{N}$, then $P\left(\lim \sup _{n \rightarrow \infty} X_{n}\right) \gg 1$. 
By again using formula (4.20), the 'if-part' of the lemma follows from this general result once we have shown that for all $n, k \in \mathbb{N}$ with $n>k$ we have

$$
\begin{aligned}
& \widetilde{m}\left(\left(\sigma^{*}\right)^{-k}\left(A_{i, k}(\epsilon)\right) \cap\left(\sigma^{*}\right)^{-n}\left(A_{i, n}(\epsilon)\right)\right) \\
& \quad \ll \widetilde{m}\left(\left(\sigma^{*}\right)^{-k}\left(A_{i, k}(\epsilon)\right)\right) \widetilde{m}\left(\left(\sigma^{*}\right)^{-n}\left(A_{i, n}(\epsilon)\right)\right) .
\end{aligned}
$$

Since $\mu^{*}$ and $\widetilde{m}$ are equivalent, and since $\mu^{*}$ is $\sigma^{*}$-invariant, it follows that in order to obtain this latter inequality it is sufficient to show that

$$
\widetilde{m}\left(A_{i, k}(\epsilon) \cap\left(\sigma^{*}\right)^{-(n-k)}\left(A_{i, n}(\epsilon)\right)\right) \ll \widetilde{m}\left(A_{i, k}(\epsilon)\right) \widetilde{m}\left(A_{i, n}(\epsilon)\right) .
$$

Since the set $A_{i, k}(\epsilon)$ can be written as a union of $S^{*}$-cylinders of length 1 , it can be written also as a union of cylinders of length $(n-k)$. If $A_{i, k}(\epsilon)=$ $\bigcup B_{k}(\epsilon)$ denotes such a representation by cylinders of length $(n-k)$, then by the $\sigma^{*}$-invariance of $\mu^{*}$ and by the Bounded Distortion Property (7) and the conformality of $m$, we have for each $\omega \in A_{i, n}(\epsilon)$ and $\tau \in B_{k}(\epsilon)$ that

$$
\begin{aligned}
& \widetilde{m}\left(\left(\sigma^{*}\right)^{-k}\left(A_{i, k}(\epsilon)\right) \cap\left(\sigma^{*}\right)^{-n}\left(A_{i, n}(\epsilon)\right)\right) \\
& \quad \asymp \widetilde{m}\left(\left(\sigma^{*}\right)^{-(n-k)}\left(A_{i, n}(\epsilon)\right) \cap B_{k}(\epsilon)\right) \\
& \left.\asymp\left|\phi_{\tau}^{\prime}(\pi(\omega))\right|^{h} \widetilde{m}\left(A_{i, n}(\epsilon)\right) \cap\left(\sigma^{*}\right)^{n-k}\left(B_{k}(\epsilon)\right)\right) .
\end{aligned}
$$

This implies that

$$
\frac{\widetilde{m}\left(\left(\sigma^{*}\right)^{-(n-k)}\left(A_{i, n}(\epsilon)\right) \cap B_{k}(\epsilon)\right)}{\widetilde{m}\left(B_{k}(\epsilon)\right)} \asymp \frac{\left|\phi_{\tau}^{\prime}(\pi(\omega))\right|^{h} \widetilde{m}\left(A_{i, n}(\epsilon)\right)}{\left|\phi_{\tau}^{\prime}(\pi(\omega))\right|^{h}}=\widetilde{m}\left(A_{i, n}(\epsilon)\right),
$$

or equivalently that

$$
\widetilde{m}\left(\left(\sigma^{*}\right)^{-(n-k)}\left(A_{i, n}(\epsilon)\right) \cap B_{k}(\epsilon)\right) \asymp \widetilde{m}\left(A_{i, n}(\epsilon)\right) \widetilde{m}\left(B_{k}(\epsilon)\right) .
$$

If in this latter inequality we sum up over all sets $B_{k}(\epsilon)$, we obtain

$$
\widetilde{m}\left(\left(\sigma^{*}\right)^{-(n-k)}\left(A_{i, n}(\epsilon)\right) \cap A_{i, k}(\epsilon)\right) \asymp \widetilde{m}\left(A_{i, n}(\epsilon)\right) \widetilde{m}\left(A_{i, k}(\epsilon)\right),
$$

which in particular gives the desired inequality.

Lemma 4.5. For $i \in \Omega$ and $\epsilon \geq 0$ we have $\widetilde{m}\left(A_{i, \infty}(\epsilon)\right)=1$.

Proof. Let $i \in \Omega$ and $\epsilon>0$ be fixed. Clearly, $\sigma^{*}\left(A_{i, \infty}(\epsilon)\right) \subset A_{i, \infty}(\epsilon)$. Hence, using the ergodicity of the map $\sigma^{*}$ and the previous lemma, the statement of the lemma follows.

Theorem 4.6 (Limit Law I). For $\widetilde{m}$-almost every $\omega \in I_{*}^{\infty}$ and for all $i \in \Omega$ we have

$$
\limsup _{n \rightarrow \infty} \frac{\log Q_{i}\left(\left(\sigma^{*}\right)^{n}(\omega)\right)}{\log n}=\frac{p_{i}}{h+(h-1) p_{i}} .
$$

Proof. In order to obtain the lower bound for the 'limsup' in the lemma, fix some $i \in \Omega$ and note that by Lemma 4.5 we have $\widetilde{m}\left(A_{i, \infty}(0)\right)=1$. If 
$\omega \in A_{i, \infty}(0)$, there exists by definition a sequence $\left(k_{j}\right)_{j \in \mathbb{N}}$ of natural numbers $k_{j}$, such that $\left(\sigma^{*}\right)^{k_{j}}(\omega) \in A_{i, k_{j}}(0)$ for all $j \in \mathbb{N}$. This implies for all $j$ that

$$
Q_{i}\left(\left(\sigma^{*}\right)^{k_{j}}(\omega)\right) \geq k_{j}^{p_{i} /\left(h+(h-1) p_{i}\right)},
$$

and hence that

$$
\limsup _{n \rightarrow \infty} \frac{\log Q_{i}\left(\left(\sigma^{*}\right)^{n}(\omega)\right)}{\log n} \geq \frac{p_{i}}{h+(h-1) p_{i}} .
$$

In order to obtain the upper bound for the 'lim sup' in the lemma, let $\epsilon<0$ and $i \in \Omega$. By Lemma 4.4, there exists a set $F_{i}(\epsilon)$ such that $\widetilde{m}\left(F_{i}(\epsilon)\right)=1$, and such that if $\omega \in F_{i}(\epsilon)$ then there exists a number $n_{0}=n_{0}(\omega) \in \mathbb{N}$ with the property that $\left(\sigma^{*}\right)^{n}(\omega) \notin A_{i, n}(\epsilon)$ for all $n \geq n_{0}$. Hence, for $\omega \in F_{i}(\epsilon)$ we have for all $n \geq n_{0}$ that

$$
\limsup _{n \rightarrow \infty} \frac{\log Q_{i}\left(\left(\sigma^{*}\right)^{n}(\omega)\right)}{\log n} \leq \frac{p_{i}}{h+(h-1) p_{i}}-\epsilon .
$$

If we put $F_{i}=\bigcap_{n \geq 1} F_{i}\left(-\frac{1}{n}\right)$, then $\widetilde{m}\left(F_{i}\right)=1$ and for each $\omega \in F_{i}$ we have

$$
\limsup _{n \rightarrow \infty} \frac{\log Q_{i}\left(\left(\sigma^{*}\right)^{n}(\omega)\right)}{\log n} \leq \frac{p_{i}}{h+(h-1) p_{i}} .
$$

Hence, for $\omega \in A_{i, \infty}(0) \cap B_{i}$ we obtain the equality stated in the theorem.

Note that if $Q_{i}(\omega)=n$, then it follows from (3.3) that $\left|x_{i}-\pi(\omega)\right| \asymp$ $(n+1)^{-1 / p_{i}}$. This now leads to our second limit law.

Theorem 4.7 (Limit Law II). For $\widetilde{m}$-almost every $\omega \in I_{*}^{\infty}$ we have for all $i \in \Omega$ that

$$
\limsup _{n \rightarrow \infty} \frac{-\log \left|\pi\left(\left(\sigma^{*}\right)^{n}(\omega)\right)-x_{i}\right|}{\log n}=\frac{1}{h+(h-1) p_{i}} .
$$

Proof. Fix $\omega \in I_{*}^{\infty}$ and $i \in \Omega$. By definition of $Q_{i}$ and using (3.3), we have for $n \in \mathbb{N}$ that

$$
\left|\pi\left(\left(\sigma^{*}\right)^{n}(\omega)\right)-x_{i}\right| \asymp\left(Q_{i}\left(\left(\sigma^{*}\right)^{n}(\omega)\right)+1\right)^{-1 / p_{i}} .
$$

Hence, it follows that

$$
\lim _{n \rightarrow \infty}\left|\frac{-\log \left|\pi\left(\left(\sigma^{*}\right)^{n}(\omega)\right)-x_{i}\right|}{\log n}-\frac{\log Q_{i}\left(\left(\sigma^{*}\right)^{n}(\omega)\right)}{p_{i} \log n}\right|=0 .
$$

Using Limit Law I, we find that, for $\widetilde{m}$-almost all $\omega \in I_{*}^{\infty}$,

$$
\begin{aligned}
\limsup _{n \rightarrow \infty} \frac{-\log \left|\pi\left(\left(\sigma^{*}\right)^{n}(\omega)\right)-x_{i}\right|}{\log n} & =\frac{1}{p_{i}} \limsup _{n \rightarrow \infty} \frac{\log Q_{i}\left(\left(\sigma^{*}\right)^{n}(\omega)\right)}{\log n} \\
& =\frac{1}{h+(h-1) p_{i}} .
\end{aligned}
$$


Since $\widetilde{m}$ is ergodic and positive on nonempty open sets, we have that $\widetilde{m}$ almost every point in $I_{*}^{\infty}$ has arbitrarily long blocks with parabolic entries only. Taking this observation into account, we now modify on a set of full measure the definition of the hyperbolic zoom $\left(r_{j}(\omega)\right)_{j}$ as follows: for a given $i \in \Omega$ we include only those elements in the hyperbolic zoom for which $n_{j}(\omega) \geq n_{j-1}(\omega)+2$ and $i(\omega, j)=i$. In other words, we consider subsequences $\left(r_{j_{k}}(\omega)\right)_{k}$ and $\left(n_{j_{k}}(\omega)\right)_{k}$ such that $n_{j_{k}}(\omega) \geq n_{j_{k}-1}(\omega)+2$ and $\omega_{n_{j_{k}-1}(\omega)}=i$. Such subsequences will be referred to as the $i$-restricted hyperbolic zoom and the $i$-restricted optimal sequence, respectively.

Theorem 4.8 (Limit Law III). For each $i \in \Omega$ the $i$-restricted optimal sequence at $\widetilde{m}$-almost every $\omega \in I_{*}^{\infty}$ has the property that

$$
\limsup _{k \rightarrow \infty} \frac{\log \left(n_{j_{k}+1}(\omega)-n_{j_{k}}(\omega)\right)}{\log j_{k}}=\frac{p_{i}}{h+(h-1) p_{i}} .
$$

Proof. Let $i \in \Omega$ and $\omega \in I_{*}^{\infty}$. Define the function $N_{n}: I_{*}^{\infty} \rightarrow \mathbb{N}$ by $\left(\sigma^{*}\right)^{n}(\omega)=\sigma^{N_{n}(\omega)}(\omega)$, for every $n \geq 1$. Then we see by induction that $N_{j}(\omega)=n_{j}(\omega)$, for all $j \in \mathbb{N}$ (this follows, since $n_{1}(\omega)=N_{1}(\omega)$ and, assuming that $n_{j}(\omega)=N_{j}(\omega)$, since $n_{j+1}(\omega)=n_{j}(\omega)+N_{1}(\omega)\left(\sigma^{n_{j}(\omega)}(\omega)\right)=$ $\left.N_{j+1}(\omega)\right)$.

Using Limit Law II and the fact that $\left|\pi\left(\sigma^{N_{j_{k}}(\omega)}(\omega)\right)-x_{i}\right| \asymp\left(N_{j_{k}+1}(\omega)-\right.$ $\left.N_{j_{k}}(\omega)\right)^{-1 / p_{i}}$, it follows that for $\widetilde{m}$-almost all $\omega$ we have

$$
\begin{aligned}
\limsup _{k \rightarrow \infty} \frac{\log \left(n_{j_{k}+1}(\omega)-n_{j_{k}}(\omega)\right)}{\log j_{k}} & =\limsup _{k \rightarrow \infty} \frac{\log \left(N_{j_{k}+1}(\omega)-N_{j_{k}}(\omega)\right)}{\log j_{k}} \\
& =\limsup _{k \rightarrow \infty} \frac{-p_{i} \log \left|\pi\left(\sigma^{N_{j_{k}}(\omega)}(\omega)\right)-x_{i}\right|}{\log j_{k}} \\
& =\limsup _{k \rightarrow \infty} \frac{-p_{i} \log \left|\pi\left(\left(\sigma^{*}\right)^{j_{k}}(\omega)\right)-x_{i}\right|}{\log j_{k}} \\
& =\frac{p_{i}}{h+(h-1) p_{i}} .
\end{aligned}
$$

Theorem 4.9 (Limit Law IV). For each $i \in \Omega$ the $i$-restricted hyperbolic zoom at $\widetilde{m}$-almost every $\omega \in I_{*}^{\infty}$ has the property that

$$
\limsup _{k \rightarrow \infty} \frac{\log \left(r_{j_{k}}(\omega) / r_{j_{k}+1}(\omega)\right)}{\log j_{k}}=\frac{1+p_{i}}{h+(h-1) p_{i}} .
$$

Proof. For $i \in \Omega$ and $\omega \in I_{*}^{\infty}$ we saw in the proof of Theorem 3.5 that

$$
\frac{r_{j_{k}}(\omega)}{r_{j_{k}+1}(\omega)} \asymp\left(n_{j_{k}+1}(\omega)-n_{j_{k}}(\omega)\right)^{\left(1+p_{i}\right) / p_{i}}
$$


for $k \in \mathbb{N}$. Combining this estimate with Limit Law III, it follows for $\widetilde{m}$ almost all $\omega \in I_{*}^{\infty}$ that

$$
\begin{aligned}
\limsup _{k \rightarrow \infty} \frac{\log \left(r_{j_{k}}(\omega) / r_{j_{k}}(\omega)\right)}{\log j_{k}} & =\limsup _{k \rightarrow \infty} \frac{1+p_{i}}{p_{i}} \frac{\log \left(n_{j_{k}+1}(\omega)-n_{j_{k}}(\omega)\right)}{\log j_{k}} \\
& =\frac{1+p_{i}}{h+(h-1) p_{i}} .
\end{aligned}
$$

The following theorem presents the main result in this section:

Theorem 4.10. (The Khintchine Limit Law for parabolic iterated function systems). The hyperbolic zoom at $\widetilde{m}$-almost every $\omega \in I_{*}^{\infty}$ satisfies

$$
\limsup _{j \rightarrow \infty} \frac{\log \left(r_{j}(\omega) / r_{j+1}(\omega)\right)}{\log \log \frac{1}{r_{j}(\omega)}}=\frac{1+p_{\max }}{h+(h-1) p_{\max }},
$$

where we have set $p_{\max }:=\max \left\{p_{i}: i \in \Omega\right\}$.

Proof. For $\widetilde{m}$-almost all $\omega \in I_{*}^{\infty}$ we have

$$
\begin{aligned}
\lim _{j \rightarrow \infty} \frac{\log r_{j}(\omega)}{j} & =\lim _{j \rightarrow \infty} \frac{\log \left|\phi_{n_{j}(\omega)}^{\prime}\left(\pi\left(\sigma^{n_{j}(\omega)}(\omega)\right)\right)\right|}{j} \\
& =\lim _{j \rightarrow \infty} \frac{\log \mid \phi_{N_{j}(\omega)}^{\prime}\left(\pi\left(\sigma^{N_{j}(\omega)}(\omega)\right) \mid\right.}{j} \\
& =\lim _{j \rightarrow \infty} \frac{\log \left|\phi_{N_{j}(\omega)}^{\prime}\left(\pi\left(\sigma^{*}\right)^{j}(\omega)\right)\right|}{j}=\chi,
\end{aligned}
$$

where the last equality follows from the Birkhoff Ergodic Theorem, using the ergodicity of the system $\left(I_{*}^{\infty}, \sigma^{*}, \mu^{*}\right)$ and the definition

$$
\chi:=\int_{I_{*}^{\infty}} \log \left|\phi_{\omega_{1}}^{\prime}\left(\pi\left(\sigma^{*}\right)(\omega)\right)\right| d m^{*}(\omega)>-\infty .
$$

Hence,

$$
\lim _{j \rightarrow \infty} \frac{\log \log \frac{1}{r_{j}(\omega)}}{\log j}=1 .
$$

The theorem follows by combining this equality with Limit Law IV and noting that

$$
\max _{i \in \Omega} \frac{1+p_{i}}{h+(h-1) p_{i}}=\frac{1+p_{\max }}{h+(h-1) p_{\max }} .
$$

Corollary 4.11. For the function $\zeta$ of the h-conformal measure $m$ (see Theorem 3.5) associated with a tame parabolic finite iterated function system satisfying (SSOSC) the following hold:

(i) For $h=1$, we have for all $\omega \in I_{*}^{\infty}$ and $0<r<\operatorname{diam}\left(I_{*}^{\infty}\right)$ that

$$
\zeta(\omega, r) \asymp 1 .
$$


(ii) For $h<1$, we have for $\widetilde{m}$-almost every $\omega \in I_{*}^{\infty}$ that

$$
\limsup _{r \rightarrow 0} \frac{\log \zeta(\omega, r)}{\log \log \frac{1}{r}}=\frac{(1-h) p_{\max }}{h+(h-1) p_{\max }} .
$$

(iii) For $h>1$, we have for $\widetilde{m}$-almost every $\omega \in I_{*}^{\infty}$ that

$$
\liminf _{r \rightarrow 0} \frac{\log \zeta(\omega, r)}{\log \log \frac{1}{r}}=\frac{(1-h) p_{\max }}{h+(h-1) p_{\max }} .
$$

Proof. Statement (i) is an immediate consequence of Theorem 3.5. In order to prove statement (ii), let $\omega \in I_{*}^{\infty}$ and $r>0$ sufficiently small be given. Without loss of generality we may assume that $r_{j+1}(\omega) \leq r<r_{j}(\omega)$ and that $\omega_{n_{j}(\omega)+1}=i$, for some $i \in \Omega$. For $r$ in this range, an elementary calculation shows that the maximal value of $\zeta(\omega, r)$ is achieved if $r$ is comparable to

$$
r_{j, \max }(\omega):=r_{j}(\omega)\left(\frac{r_{j+1}(\omega)}{r_{j}(\omega)}\right)^{1 /\left(1+p_{i}\right)} .
$$

For this value of $r$ we have

$$
\zeta\left(\omega, r_{j, \max }(\omega)\right) \asymp\left(\frac{r_{j}(\omega)}{r_{j+1}(\omega)}\right)^{(1-h) p_{i} /\left(1+p_{i}\right)} .
$$

As we have seen above in the proof of the Khintchine law, for $\widetilde{m}$-almost all $\omega \in I_{*}^{\infty}$ it is sufficient to restrict the discussion to those indices $j$ for which $\omega_{n_{j}(\omega)}=i$, with $p_{i}=p_{\max }$. It follows that for all $\epsilon>0$ and for $m$-almost all $\omega \in I_{*}^{\infty}$ we eventually have

$\frac{(1-\epsilon)\left(1+p_{i}\right)}{h+(h-1) p_{i}} \log \log \frac{1}{r_{j}(\omega)} \leq_{\text {i.o. }} \log \frac{r_{j}(\omega)}{r_{j+1}(\omega)} \leq \frac{(1+\epsilon)\left(1+p_{i}\right)}{h+(h-1) p_{i}} \log \log \frac{1}{r_{j}(\omega)}$ (where ' $\leq_{\text {i.o.' }}$ indicates that the inequality holds 'infinitely often', i.e., for some infinite sequence of values of $j$ ). Hence, the estimate above implies that

$$
\left(\log \frac{1}{r_{j}(\omega)}\right)^{\frac{(1-\epsilon)(1-h) p_{\max }}{h+(h-1) p_{\max }}} \ll_{\text {i.o. }} \zeta\left(\omega, r_{j, \max }(\omega)\right) \ll\left(\log \frac{1}{r_{j}(\omega)}\right)^{\frac{(1+\epsilon)(1-h) p_{\max }}{h+(h-1) p_{\max }}} .
$$

This proves statement (ii) in the corollary. Statement (iii) follows from a similar argument, and we omit its proof.

We are now in the position to derive a refinement of the description of the geometric nature of the $h$-conformal measure given in Theorem 3.8 . Namely, using the latter corollary, we have the following statements concerning its relationship to the packing measure $\mathrm{P}_{\psi_{\lambda}}$ and Hausdorff measure $\mathrm{H}_{\psi_{\lambda}}$ with respect to the dimension function $\psi_{\lambda}$. Here, the function $\psi_{\lambda}$ is given for $\lambda \in \mathbb{R}$ and positive $r$ by

$$
\psi_{\lambda}(r):=r^{h}\left(\log \frac{1}{r}\right)^{(1+\lambda)(1-h) p_{\max } /\left(h+(h-1) p_{\max }\right)} .
$$


Corollary 4.12. If $S$ is a tame finite parabolic iterated function system satisfying (SSOSC), we have the following table:

\begin{tabular}{|c|c|c|}
\hline$\lambda$ vs. $h$ & $h<1$ & $h>1$ \\
\hline$\lambda>0$ & $m \ll \mathcal{H}_{\psi_{\lambda}}$ and $\mathcal{H}_{\psi_{\lambda}}(J)=\infty$ & $\exists E_{\lambda}$ s.t. $m\left(E_{\lambda}\right)=1, \mathcal{P}_{\psi_{\lambda}}\left(E_{\lambda}\right)=0$ \\
\hline$\lambda \leq 0$ & $\exists F_{\lambda}$ s.t. $m\left(F_{\lambda}\right)=1, \mathcal{H}_{\psi_{\lambda}}\left(F_{\lambda}\right)=0$ & $m \ll \mathcal{P}_{\psi_{\lambda}}$ and $\mathcal{P}_{\psi_{\lambda}}(J)=\infty$ \\
\hline
\end{tabular}

The symbol ' $\ll$ ' indicates absolute continuity between two measures.

Acknowledgements. We thank the Stochastic Institute at the University of Göttingen, the Mathematics Department at the University of Warwick and IMPA in Rio de Janeiro for their warm hospitality and excellent working conditions when writing this paper.

\section{References}

[B] A.S. Besicovitch, Sets of fractional dimension (IV): On rational approximation to real numbers, Jour. London Math. Soc., 9 (1934), 126-131, Zbl 0009.05301.

[HV] R. Hill and S.L. Velani, The Jarnik-Besicovitch Theorem for geometrically finite Kleinian groups, Proc. London Math. Soc. (3), 77 (1998), 524-550, MR 1643409 (99f:11100), Zbl 0924.11063.

[J] V. Jarník, Diophantische Approximationen und Hausdorffsches Maß, Mathematicheskii Sbornik, 36 (1929), 371-382, Zbl 55.0719.01.

[K] A. Khintchine, Continued Fractions, Noordhoff, Groningen, 1963, MR 0161834 (28 \#5038). Translation of Tspenye dobni, 3rd ed., Nauka, Moscow, 1961.

[MU1] R. D. Mauldin and M. Urbański, Parabolic iterated function systems, Ergod. Th. \& Dynam. Sys., 20 (2000), 1423-1447, MR 1786722 (2001m:37047), Zbl 0982.37045.

[MU2] R. D. Mauldin, M. Urbański, Fractal measures for parabolic IFS, Adv. in Math., 168 (2002), 225-253, MR 1912133 (2003e:28023), Zbl 1013.28007.

[MU3] R. D. Mauldin, M. Urbański, Graph Directed Markov Systems: Geometry and Dynamics of Limit Sets, Cambridge Tracts in Mathematics, 148 Cambridge University Press, Cambridge, 2003, MR 2003772, Zbl 1033.37025.

[S1] B.O. Stratmann, Fractal dimensions for Jarnik limit sets of geometrically finite Kleinian groups; the semi-classical approach, Ark. Math., 33 (1995), 385-403, MR 1373031 (97a:30056), Zbl 0851.30027.

[S2] B.O. Stratmann, Weak singularity spectra of the Patterson measure for geometrically finite Kleinian groups with parabolic elements, Michigan Math. J., 46 (1999), 573-587, MR 1721571 (2001a:37059), Zbl 0961.30033.

[S3] B.O. Stratmann, Multiple fractal aspects of conformal measures; a survey, in 'Workshop on Fractals and Dynamics', eds. M. Denker, S.-M. Heinemann and B.O. Stratmann, Math. Gottingensis, Heft 5 (1997), 65-71.

[SU1] B.O. Stratmann and M. Urbański, Jarnik and Julia; a Diophantine analysis for parabolic rational maps for geometrically finite Kleinian groups with parabolic elements, Math. Scan., 91 (2002), 27-54, MR 1917680, Zbl 1019.37028. 
[SU2] B.O. Stratmann, M. Urbański, The geometry of conformal measures for parabolic rational maps, Math. Proc. Cambridge Phil. Soc., 128 (2000), 141-156, MR 1724435 (2000i:37066), Zbl 0974.37029.

[SV] B.O. Stratmann and S. Velani, The Patterson measure for geometrically finite groups with parabolic elements, new and old, Proc. London Math. Soc. (2), 71 (1995), 197-220, MR 1327939 (97f:58023), Zbl 0821.58026.

[Su] D. Sullivan, Disjoint spheres, approximation by imaginary quadratic numbers, and the logarithm law for geodesics, Acta Math., 149 (1983), 215-237, MR 0688349 (84j:58097), Zbl 0517.58028.

[U1] M. Urbański, Rigidity of multi-dimensional conformal iterated function systems, Nonlinearity, 14 (2001), 1593-1610, MR 1867094 (2003c:37031), Zbl 1001.37023.

[U2] M Urbański, Parabolic Cantor sets, Fund. Math., 151 (1996), 241-277, MR 1424576 (98f:58076), Zbl 0895.58036.

Received January 22, 2002. The research of the second author was supported in part by the NSF Grant DMS 9801583.

Mathematical Institute

UNIVERSITY OF ST ANDREWS

ST ANDREWS KY16 9SS

SCOTLAND

E-mail address: bos@maths.st-and.ac.uk

Department of Mathematics

University of North TeXas

DENTON TX 76203-1430

E-mail address: urbanski@dynamics.math.unt.edu 